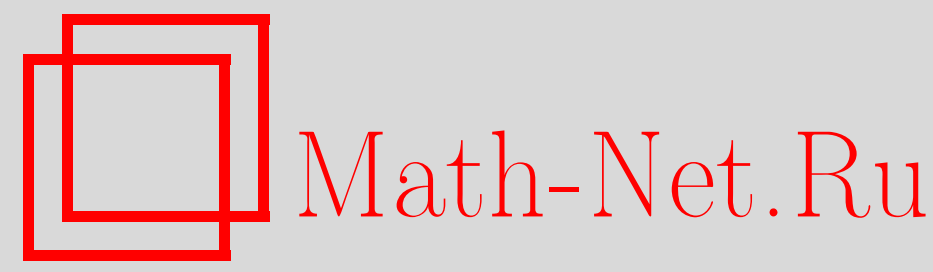

В. В. Никулин, Группы отражений в пространствах Лобачевского и тождество для знаменателя лоренцевых алгебр Каца-Муди, Изв. РАН. Сер. матем., 1996, том 60, выпуск 2, 73-106

DOI: https://doi.org/10.4213/im72

Использование Общероссийского математического портала Math-Net.Ru подразумевает, что вы прочитали и согласны с пользовательским соглашением

http://www.mathnet.ru/rus/agreement

Параметры загрузки:

IP : 54.198 .67 .100

26 апреля 2023 г., 16:36:16 
УДК $512.75+512.817+512.818$

\section{В.В. Никулин}

\section{Группы отражений в пространствах Лобачевского и тождество для знаменателя лоренцевых алгебр Каца-Муди}

\footnotetext{
Строится некоторая теория лоренцевых алгебр Каца-Муди, основанная на теории групп отражений в пространствах Лобачевского и недавних результатах Р. Борчердса.

Библиография: 35 наименований.
}

\section{Введение}

В [25] было показано, что множество мнимых корней конечно порожденной симметризуемой алгебры Каца-Муди "хорошо себя ведет", если и только если эта алгебра Ли принадлежит одному из четырех классов: конечномерных, аффинных, гиперболических ранга два, гиперболических арифметического типа. Здесь гиперболический арифметический тип означает, что группа Вейля является группой отражений в пространстве Лобачевского размерности $\geqslant 2$ с фундаментальньм многогранником конечного объема. Данная работа естественным образом продолжает это исследование.

Мы рассматриваем некоторую гиперболическую (т.е. сигнатуры $(n, 1))$ целочисленную симметрическую билинейную форму $S: M \times M \rightarrow \mathbb{Z}$ (т.е. гиперболическую решетку), группу отражений $W \subset W(S)$, фундаментальньй многогранник $\mathcal{M}$ группы $W$ и допустимое (соответствующее скручивающим коэффициентам) множество $P(\mathcal{M}) \subset M$ векторов, ортогональных граням $\mathcal{M}$ (простые вешественные корни). По этим данным строится соответствуюшая лоренцева алгебра Каца-Муди $\mathfrak{g}=\mathfrak{g}^{\prime \prime}(A(S, W, P(\mathcal{M})))$, которая градуирована решеткой $M$ (см. пп. 2.1 и 2.2).

Мы показываем, что для алгебры $\mathfrak{g}=\mathfrak{g}^{\prime \prime}(A(S, W, P(\mathcal{M})))$ мнимые корни "хорошо себя ведут", ее формула для знаменателя определена в естественной области и имеет хорошие автоморфные свойства, если и только если эта алгебра имеет так называемый ограниченный арифметический тип (см. п. 2.2). Это означает, что полупрямое произведение $W . A(P(\mathcal{M}))$ имеет конечный индекс в $O(S)$. Здесь

$$
A(P(\mathcal{M}))=\left\{g \in O_{+}(S) \mid g(P(\mathcal{M}))=P(\mathcal{M})\right\}
$$

Автор благодарен за финансовую поддержку Российскому Фонду фундаментальных исследований; Фонду Сороса, грант МI6000; Фонду Сороса и Российского правительства, грант MI6300; Американскому Математическому обществу; SFB 170 "Geometrie und Analysis" at Göttingen. 
- “группа симметрий” фундаментального многогранника. Мы показываем, что каждая конечно порожденная (т. е. $P\left(\mathcal{M}_{1}\right)$ конечно) лоренцева алгебра Каца-Муди $\mathfrak{g}^{\prime \prime}\left(A\left(S, W_{1}, P\left(\mathcal{M}_{1}\right)\right)\right)$ вкладывается в лоренцеву алгебру Каца-Муди $\mathfrak{g}^{\prime \prime}(A(S, W, P(\mathcal{M})))$ ограниченного арифметического типа и с той же решеткой $S$ (см. п. 2.2). Поэтому естественно изучать лоренцевы алгебры Каца-Муди ограниченного арифметического типа.

Формула для знаменателя лоренцевой алгебры Каца-Муди ограниченного арифметического типа имеет особенно хорошие автоморфные свойства, если и только если эта алгебра имеет решеточный вектор Вейля $\rho \in M \otimes \mathbb{Q}$ (см. п. 2.3, а также п. 2.4). Решеточный вектор Вейля - это такой элемент $\rho \in M \otimes \mathbb{Q}$, что

$$
S(\rho, \alpha)=-S(\alpha, \alpha) / 2 \quad \text { для любого } \alpha \in P(\mathcal{M})
$$

Лоренцева алгебра Каца-Муди с обобшенным решеточным вектором Вейля $\rho$ (см. определение 1.4.9) называется әллиптической, если она имеет ограниченный арифметический тип и $S(\rho, \rho)<0$; и называется параболической, если она имеет ограниченный арифметический тип и $S(\rho, \rho)=0$ и не имеет обобщенного вектора Вейля с отрицательным квадратом. Эллиптичность эквивалентна конечности индекса $[O(S): W]$. Параболичность эквивалентна ограниченному арифметическому типу и существованию $0 \neq c \in M$ такого, что $S(c, c)=0$ и $g(c)=c$ для любого $g \in A(P(\mathcal{M}))$, где $A(P(\mathcal{M}))$ бесконечна. Соответствующая решетка $S$ называется әллиптической рефлективной и параболической рефлективной соответственно.

Используя и обобшая наши результаты и результаты Э.Б. Винберга по группам отражений в пространстах Лобачевского, мы показываем, что множества примитивных эллиптических и параболических рефлективных решеток $S$ ранга rk $S \geqslant 3$ конечны (см. п. 1.1). Для эллиптического случая это было известно почти 15 лет назад. Таким образом, мы обобщаем данньй результат конечности на параболический случай. Удивительным образом в точности тот же метод, который был использован для эллиптического случая, работает и для параболического. Это показывает, что эти два случая очень близки и метод, использованный для эллиптического случая, очень естествен.

Мы применяем основной геометрический факт, которьй был использован для получения вышеприведенных результатов конечности, чтобы показать, что множество эллиптических лоренцевых алгебр Каца-Муди $\mathfrak{g}^{\prime \prime}(A(S, W, P(\mathcal{M})))$ с решеточным вектором Вейля $\rho$ конечно для $\operatorname{rk} S \geqslant 3$. Для параболического случая мы получаем тот же самый результат при дополнительном предположении, что индекс $\left[O(S)_{\rho}: A(P(\mathcal{M}))\right]<D$ для некоторой зафиксированной константы $D>0$. Здесь $O(S)_{\rho}$ - стационарная подгруппа $\rho$ (см. п. 1.3). Пример 1.3.4 показывает, что без этого дополнительного условия конечность может не иметь место для параболического случая.

Наконец, в п. 2.4 мы рассматриваем связь наших результатов с недавними результатами Р. Борчердса.

Данная статья написана в течение моего пребывания в МИАН им. В.А. Стеклова и в Математическом институте университета г. Гёттингена (январь-март 1995 г.). Я благодарен этим институтам за гостеприимство. 
Я благодарен Э.Б. Винбергу за очень важные замечания. Я благодарен И.Р. Шафаревичу за его интерес и поддержку данных моих исследований.

Предварительный вариант данной статьи опубликован в качестве препринта [26].

\section{§1. Некоторые результаты по группам отражений целочисленных гиперболических решеток}

\section{1. Эллиптические и параболические рефлективные решетки и} группы отражений. Результаты данного пункта дополняют результаты работ автора [19], [20] и работы Э.Б. Винберга [32]. В [19] и [20] мы рассматривали гиперболические решетки сигнатуры $(1, n)$ и обозначали пространство и форму одной буквой $S$. Здесь мы будем использовать для обозначения формы две буквы: $S$ и $M$. Также мы придерживаемся сигнатуры $(n, 1)$ для гиперболической формы. Эти обозначения стандартны для теории алгебр Ли.

Здесь мы обобшаем результаты вьшеупомянутых работ на "групшы отражений с каспом".

Пусть

$$
S: M \times M \rightarrow \mathbb{Z}
$$

- гиперболическая (т.е. сигнатуры $(n, 1))$ целочисленная симметрическая билинейная форма над $\mathbb{Z}$. Здесь $M-$ свободный $\mathbb{Z}$-модуль конечного ранга. Для краткости мы будем называть $S$ әиперболической решеткой. Мы рассматриваем соответствующий конус

$$
V(S)=\{x \in M \otimes \mathbb{R} \mid S(x, x)<0\},
$$

выбираем полу $V^{+}(S)$ этого конуса и рассматриваем соответствуюшее пространство Лобачевского $\mathcal{L}(S)=V^{+}(S) / \mathbb{R}_{++}$, где ++ обозначает множество положительных чисел. Таким образом, точкой $\mathcal{L}(S)$ является луч $\mathbb{R}_{++} x$, где $x \in V^{+}(S)$. Расстояние $\rho$ в $\mathcal{L}(S)$ задается формулой

$$
\operatorname{ch} \rho\left(\mathbb{R}_{++} x, \mathbb{R}_{++} y\right)=-S(x, y) / \sqrt{S(x, x) S(y, y)}, \quad x, y \in V^{+}(S) .
$$

При таком определении расстояния кривизна равна -1 . Каждый элемент $\delta \in$ $M \otimes \mathbb{R}$ с положительным квадратом $S(\delta, \delta)>0$ определяет полупространство

$$
\mathcal{H}_{\delta}^{+}=\left\{\mathbb{R}_{++} x \in \mathcal{L}(S) \mid S(x, \delta) \leqslant 0\right\},
$$

ограниченное гиперплоскостью

$$
\mathcal{H}_{\delta}=\left\{\mathbb{R}_{++} x \in \mathcal{L}(S) \mid S(x, \delta)=0\right\} .
$$

Элемент $\delta \in M \otimes \mathbb{R}$ определяется полупространством $\mathcal{H}_{\delta}^{+}$(соответственно гиперплоскостью $\mathcal{H}_{\delta}$ ) однозначно с точностью до умножения на элементы $\mathbb{R}_{++}($соответственно на элементы множества $\mathbb{R}^{*}$ ненулевых действительных чисел). Такой элемент $\delta$ называется ортогональным полупространству $\mathcal{H}_{\delta}^{+}$(соответственно гиперплоскости $\left.\mathcal{H}_{\delta}\right)$. 
Пусть $O_{+}(S)$ - подгруппа индекса 2 групшы $O(S)$, которая сохраняет полу $V^{+}(S)$ конуса. Хорошо известно (это легкое следствие общей теории арифметических групп), что $O_{+}(S)$ дискретна в $\mathcal{L}(S)$ и имеет фундаментальную область конечного объема. Если $\phi \in O_{+}(S)$ задает отражение $\mathcal{L}(S)$ в гиперплоскости, то $\phi=s_{\delta}$ для некоторого $\delta \in M$ с квадратом $S(\delta, \delta)>0$. Здесь

$$
s_{\delta}(x)=x-(2 S(x, \delta) / S(\delta, \delta)) \delta, \quad x \in S,
$$

и $s_{\delta} \in O(S)$, если и только если

$$
(2 S(M, \delta) / S(\delta, \delta)) \delta \subset M
$$

В частности, если $\delta$ примитивен в $M$, то это эквивалентно

$$
S(\delta, \delta) \mid 2 S(M, \delta)
$$

Очевидно, $s_{\delta}$ - отражение в гиперплоскости $\mathcal{H}_{\delta}$, которая ортогональна $\delta$. Данное отражение меняет местами полупространства $\mathcal{H}_{\delta}^{+}$и $\mathcal{H}_{-\delta}^{+}$. Автоморфизм $s_{\delta} \in O(S)$ называется отражением решетки $S$. Подгруппа группы $O(S)$ (или соответствуюшая дискретная группа движений пространства Лобачевского $\mathcal{L}(S))$, порожденная некоторым множеством отражений, называется группой отражсений решетки $S$.

Обозначим через $W(S)$ подгруппу $O_{+}(S)$, порожденную всеми отражениями $S$. Решетка $S$ называется рефлективной, если индекс $[O(S): W(S)]$ конечен. Это эквивалентно тому, что $W(S)$ имеет фундаментальньй многогранник конечного объема в $\mathcal{L}(S)$. Э. Б. Винберг, в частности, показал [30], что любая арифметическая группа отражений $W$ с полем определения $\mathbb{Q}$ в пространстве Лобачевского является подгруппой $W \subset W(S)$ конечного индекса для одной из рефлективных гиперболических решеток $S$. Для $m \in \mathbb{Q}$ обозначим через $S(m)$ решетку, которая получается умножением формы $S$ на $m$. Очевидно, $S(m)$ и $S$ рефлективны одновременно.

15 лет назад была доказана

Теорема 1.1.1 [19, приложение, теорема 1], [20, теорема 5.2.1]. Для фиксированного $\mathrm{rk} S \geqslant 3$ множество рефлективных гиперболических решеток $S$ является конечным с точностью до подобия $S \mapsto S(m)$ и изоморфизма.

Доказательство было основано на одном чисто геометрическом результате о выпуклых многогранниках конечного объема в пространствах Лобачевского, который мы хотим сформулировать.

Выпуклый многогранник $\mathcal{M}$ в пространстве Лобачевского $\mathcal{L}(S)$ является пересечением:

$$
\mathcal{M}=\bigcap_{\delta \in P(\mathcal{M})} \mathcal{H}_{\delta}^{+}
$$

нескольких полупространств, ортогональных элементам $\delta \in M \otimes \mathbb{R}$ с $S(\delta, \delta)>0$. Мы предполагаем, что $\mathcal{M}$ является локально конечньм в $\mathcal{L}(S)$. Тогда минимальное множество $P(\mathcal{M})$ в (1.1.9) определено канонически с точностью до умножения 
его элементов на положительные действительные числа и называется множеством векторов, ортогональных граням $\mathcal{M}$ ( и направленных наружу), или, кратко, ортогональных $\mathcal{M}$. Ниже всегда предполагается, что $P(\mathcal{M})$ обладает этим свойством. Многогранник $\mathcal{M}$ называется невыро жденным, если он содержит непустое открытое подмножество из $\mathcal{L}(S)$. Невырожденный многогранник $\mathcal{M}$ называется әллиптическим (эквивалентно имеет конечный объем), если он является выпуклой оболочкой конечного числа точек из $\mathcal{L}(S)$ или из бесконечности $\mathcal{L}(S)$. Тогда $P(\mathcal{M})$ конечно. Доказательство теоремы 1.1 .1 основано на следуюшем результате.

ТЕОРема 1.1.1' [19, приложение, теорема 1]. Пусть $\mathcal{M}$ - әллиптический (әквивалентно конечного обвема) невырожденный выпуклый многогранник в пространстве Лобачевского $\mathcal{L}(S)$ размерности $\operatorname{dim} \mathcal{L}(S)=n \geqslant 2$.

Тогда найдутся әлементы $\delta_{1}, \ldots, \delta_{n+1} \in P(\mathcal{M})$ со следующими свойства$\mathcal{M u}$ :

(a) $\operatorname{rk}\left[\delta_{1}, \ldots, \delta_{n+1}\right]=n+1$;

(b) диаграмма Грама әлементов $\delta_{1}, \ldots, \delta_{n+1}$ связна (т.е. множество $\left\{\delta_{1}, \ldots, \delta_{n+1}\right\}$ нельзя разбить на два непустых взаимно ортогональных подмножества);

(с) $-2 \leqslant-2 S\left(\delta_{i}, \delta_{j}\right) / \sqrt{S\left(\delta_{i}, \delta_{i}\right) S\left(\delta_{j}, \delta_{j}\right)}<62$ (другими словами, мы имеем неравенство $-2 \leqslant-S\left(e_{i}, e_{j}\right)<62$ при номализации әлементов $e_{i} \in P(\mathcal{M})$ условием, что $\left.S\left(e_{i}, e_{i}\right)=2\right), 1 \leqslant i, j \leqslant n+1$.

Для доказательства теоремы 1.1.1 нужно применить теорему $1.1 .1^{\prime}$ к фундаментальному многограннику $\mathcal{M}$ группы $W$ и элементам $P(\mathcal{M})$ из решетки $M$ (см. [20, теорема 5.2.1]).

Далее мы называем группы отражений $W \subset O(S)$ конечного индекса и соответствуюшие гиперболические решетки $S$ также эллиптическими группами отражений и әллиптическими рефлективными решетками.

Мы хотим обобщить данные результаты по эллиптическим группам отражений и эллиптическим рефлективным решеткам на следующую ситуацию.

Пусть $S$ - гиперболическая решетка и $W \subset W(S)$ - группа отражений. Пусть $\mathcal{M}$ - фундаментальный многогранник группы $W$. Пусть

$$
A(\mathcal{M})=\left\{\phi \in O_{+}(S) \mid \phi(\mathcal{M})=\mathcal{M}\right\}
$$

- группа симметрий $\mathcal{M}$. Очевидно, тогда полупрямое произведение $W . A(\mathcal{M})$ является подгруппой в $O_{+}(S)$, где $W$ - нормальная подгруппа в $W \cdot A(\mathcal{M})$.

ОПРЕДЕЛЕНИЕ 1.1.2. Группа отражений $W \subset W(S)$ называется параболической, если группа $A(\mathcal{M})$ бесконечна, но имеет конечный индекс в $O(S) / W$ (это значит, что $W . A(\mathcal{M}) \subset O_{+}(S)$ имеет конечный индекс) и существует такой элемент $0 \neq c \in M$ с квадратом $S(c, c) \leqslant 0$, что $\phi(c)=c$ для каждого $\phi \in A(\mathcal{M})$. Легко видеть, что тогда (заменяя $c$ на $-c$, если необходимо) $S(c, c)=0$ и $\mathbb{R}_{++} c \in \mathcal{M}$ (эквивалентно $S(c, P(\mathcal{M})) \leqslant 0$ ). Мы назьваем примитивный элемент $c \in M$ (или точку $\mathbb{R}_{++} c$ на бесконечности $\left.\mathcal{L}(S)\right)$, который удовлетворяет этому условию, каспомгруппы $W$. Легко видеть, что касп $c$ (и точка $\mathbb{R}_{++} c$ ) единствен. Гиперболическая решетка $S$ назьвается параболической рефлективной, если $O_{+}(S)$ содержит параболическую группу отражений $W$. 
Мы хотим показать, что теорема 1.1.1 имеет место также для параболических реффлективных решеток $S$.

ТЕОРема 1.1.3. Для фиксированного $\mathrm{rk} S \geqslant 3$ множсество параболических рефлективных решеток $S$ конечно $c$ точностью до подобия $S \mapsto S(m) u$ изоморфизма.

ДокаЗАТЕльство. Докажем аналог теоремы 1.1.1' для подходящих “параболических многогранников". Фиксируем точку $O=\mathbb{R}_{++} c$ на бесконечности пространства $\mathcal{L}(S)$. Таким образом, $c \in S \otimes \mathbb{R}, S(c, c)=0$ и $S\left(c, V^{+}(S)\right)<0$.

Напомним, что орисфера $\mathcal{E}_{O}$ с центром $O$ - это множество прямых в $\mathcal{L}(S)$, содержащих $O$. Прямая $l=O \mathbb{R}_{++} h \in \mathcal{E}_{O}, \mathbb{R}_{++} h \in \mathcal{L}(S)$, - это множество $l=\left\{\mathbb{R}_{++}(t c+h) \mid t \in \mathbb{R}\right.$ и $\left.S(t c+h, t c+h)<0\right\}$. Зафиксируем константу $R>0$. Тогда существует единственная точка $\mathbb{R}_{++} h \in l$ такая, что $S(h, c)=-R$ и $S(h, h)=-1$. Пусть $l_{1}, l_{2} \in \mathcal{E}_{O}$ и $h_{1}, h_{2}$ - соответствуюшие элементы, определенные выше. Пусть

$$
\rho\left(l_{1}, l_{2}\right)=\sqrt{S\left(h_{1}-h_{2}, h_{1}-h_{2}\right)}
$$

Орисфера $\mathcal{E}_{O}$ с данным расстоянием является аффинным евклидовым пространством. При изменении $R$ расстояние $\rho$ умножается на константу. Множество

$$
\mathcal{E}_{O, R}=\left\{\mathbb{R}_{++} h \in \mathcal{L}(S) \mid S(h, c)=-R \quad \text { и } S(h, h)=-1\right\} \cup\{O\}
$$

является сферой в $\overline{\mathcal{L}}(S)$, касающейся $\mathcal{L}(S)_{\infty}$ в точке $O$. Кроме того, сфера $\mathcal{E}_{O, R}$ ортогональна каждой прямой $l \in \mathcal{E}_{O}$ в соответствующей $l$ точке $\mathbb{R}_{++} h, h \in \mathcal{E}_{O, R}$. Расстояние в $\mathcal{L}(S)$ индуцирует евклидово расстояние в $\mathcal{E}_{O, R}$, подобное расстоянию (1.1.11). Множество $\mathcal{E}_{O, R}$ отождествляется с $\mathcal{E}_{O}$ и также называется орисферой.

Пусть $K \subset \mathcal{E}_{O}$. Множество

$$
C_{K}=\bigcup_{l \in K} l
$$

называется конусом с вершиной $O$ и основанием $K$.

Невырожденный локально конечный многогранник $\mathcal{M}$ в $\mathcal{L}(S)$ называется параболическим (относительно точки $O \in \mathcal{L}(S)_{\infty}$ ), если выполнены следующие условия:

1) $\mathcal{M}$ конечен в точке $O$, т.е. множество $\{\delta \in P(\mathcal{M}) \mid S(c, \delta)=0\}$ конечно;

2 ) для любого эллиптического многогранника $\mathcal{N} \subset \mathcal{E}_{O}$ (т.е. $\mathcal{N}$ является выпуклой оболочкой конечного множества точек в $\left.\mathcal{E}_{O}\right)$ многогранник $\mathcal{M} \cap C_{\mathcal{N}}$ эллиптичен.

Параболический многогранник $\mathcal{M}$ называется ограниченным параболическим, если множество

$$
r(\mathcal{M})=\{-S(c, \delta / \sqrt{S(\delta, \delta)}) \mid \delta \in P(\mathcal{M})\}
$$

конечно. Геометрически это означает, что все гиперплоскости $\mathcal{H}_{\delta}, \delta \in P(\mathcal{M})$, касаются конечного множества орисфер с центром в $O$. 
Tеорема 1.1.3'. Теорема $1.1 .1^{\prime}$ имеет место также для ограниченных параболических многогранников $\mathcal{M}$ в пространствах Лобачевского $\mathcal{L}(S)$ размерности $\operatorname{dim} \mathcal{L}(S)=n \geqslant 2$. Таким образом, найдутся әлементы $\delta_{1}, \ldots, \delta_{n+1} \in P(\mathcal{M})$ со следующими свойствами:

(a) $\operatorname{rk}\left[\delta_{1}, \ldots, \delta_{n+1}\right]=n+1$

(b) диаграмма Грама әлементов $\delta_{1}, \ldots, \delta_{n+1}$ связна (т.е. множество $\left\{\delta_{1}, \ldots, \delta_{n+1}\right\}$ нельзя разбить на два непустых взаимно ортогональных подмножества);

(с) $-2 \leqslant-2 S\left(\delta_{i}, \delta_{j}\right) / \sqrt{S\left(\delta_{i}, \delta_{i}\right) S\left(\delta_{j}, \delta_{j}\right)} \leqslant 62$ (другими словами, мы имеем неравенство $-2 \leqslant-S\left(e_{i}, e_{j}\right) \leqslant 62$ при нормализачии әлементов $e_{i} \in P(\mathcal{M})$ условием, что $\left.S\left(e_{i}, e_{i}\right)=2\right), 1 \leqslant i, j \leqslant n+1$. (В отличие от теоремы 1.1.1', справа стоит нестрогое неравенство $\leqslant 62$.)

Фундаментальный многогранник $\mathcal{M}(W)$ параболической группь отражений $W \subset W(S)$ гиперболической решетки $S$ является ограниченным параболическим относительно каспа $\mathbb{R}_{++}$с группь $W$, и утверждения (а)-(c) имеют место для $\mathcal{M}(W)$.

ДокАЗАТЕЛЬСТво. При доказательстве мы нормализуем ортогональные векторы $\delta \in P(\mathcal{M})$ многогранника $\mathcal{M}$ условием $S(\delta, \delta)=2$. Мы нормализуем этим же условием ортогональные векторы ко всем гиперплоскостям и полупространствам в рассуждениях, приведенных ниже.

Для доказательства теоремы 1.1.1' в [19, приложение] фиксировалась точка $O$ внутри многогранника $\mathcal{M}$ и использовалась следуюшая формула для $S\left(\delta_{1}, \delta_{2}\right)$ двух элементов $\delta_{1}, \delta_{2} \in P(\mathcal{M})$ с $S\left(\delta_{1}, \delta_{1}\right)=S\left(\delta_{2}, \delta_{2}\right)=2$ (см. [19, приложение, формула (2.1)]):

$$
-S\left(\delta_{1}, \delta_{2}\right)=4 \frac{\sin \frac{\theta_{1}+\theta_{12}}{2} \sin \frac{\theta_{2}+\theta_{12}}{2}}{\sin \frac{\theta_{1}}{2} \sin \frac{\theta_{2}}{2}}-2 .
$$

Здесь $\theta_{1}$ и $\theta_{2}-$ растворы (или углы) конусов с вершиной $O$ и основаниями $\mathcal{H}_{\delta_{1}}$ и $\mathcal{H}_{\delta_{2}}$ соответственно. Для непересекающихся гиперплоскостей $\mathcal{H}_{\delta_{1}}$ и $\mathcal{H}_{\delta_{2}}$ величина $\theta_{12}$ - раствор конуса с вершиной $O$, пересекающего два предыдущих конуса и имеющего наименьший раствор (это наименьший конус, касающийся этих двух конусов). Здесь конусы и углы берутся в смысле геометрии Лобачевского. Используя аналитическое продолжение, легко обобшить эту формулу на произвольные элементы $\delta_{1}, \delta_{2}$ с $S\left(\delta_{1}, \delta_{1}\right)=S\left(\delta_{2}, \delta_{2}\right)=2$. Следует рассмотреть плоское сечение, содержашее $O$ и ортогональное гиперплоскостям $\mathcal{H}_{\delta_{1}}$ и $\mathcal{H}_{\delta_{2}}$, и ввести подходящую ориентацию плоских углов в сечении.

Для доказательства теоремы $1.1 .3^{\prime}$ мы используем подобную формулу. Предположим, что многогранник $\mathcal{M}$ является ограниченным параболическим относительно точки $O=\mathbb{R}_{++} c$ на бесконечности. Для элемента $\delta \in M \otimes \mathbb{R}$ с $S(\delta, \delta)=2$ мы вводим "угол" $\theta(\delta)=-1 / S(c, \delta)$. Имеет место следуюший аналог формулы (1.1.15):

$$
-S\left(\delta_{1}, \delta_{2}\right)=4 \frac{\left(\theta\left(\delta_{1}\right)+\theta\left(\delta_{12}\right)\right)\left(\theta\left(\delta_{2}\right)+\theta\left(\delta_{12}\right)\right)}{\theta\left(\delta_{1}\right) \theta\left(\delta_{2}\right)}-2 .
$$

Здесь, как и выше, для $\delta_{1}, \delta_{2} \in P(\mathcal{M})$ с $S\left(\delta_{1}, \delta_{1}\right)=S\left(\delta_{2}, \delta_{2}\right)=2$ и непересекающимися гиперплоскостями $\mathcal{H}_{\delta_{1}}, \mathcal{H}_{\delta_{2}}$ элемент $\delta_{12} \in M \otimes \mathbb{R}$ ортогонален гиперплос- 
кости $\mathcal{H}_{\delta_{12}}$, имеет $S\left(\delta_{12}, \delta_{12}\right)=2$ и $S\left(c, \delta_{12}\right)<0$, пересекает $\mathcal{H}_{\delta_{1}}$ и $\mathcal{H}_{\delta_{2}}$ только по бесконечно удаленным точкам и имеет минимальньй "угол" $\theta\left(\delta_{12}\right)$.

Здесь $\theta(\delta)$ ведет себя подобно величине угла с вершиной $O$ и основанием $\mathcal{H}_{\delta}$. Если $A, B, C, O$ - четыре последовательные вершины на бесконечности выпуклого четырехугольника на плоскости и $e_{1}, e_{2}, e_{3}$ ортогональны прямым $A B, B C$ и $A C$ соответственно, имеют $S\left(e_{1}, e_{1}\right)=S\left(e_{2}, e_{2}\right)=S\left(e_{3}, e_{3}\right)=2$ и $-S\left(c, e_{1}\right)>0$, $-S\left(c, e_{2}\right)>0$ и $-S\left(c, e_{3}\right)>0$, то

$$
\theta\left(e_{3}\right)=\theta\left(e_{1}\right)+\theta\left(e_{2}\right) .
$$

Формулы (1.1.15)-(1.1.17) - это формулы элементарной аналитической 2-мерной геометрии Лобачевского, если рассмотреть плоскость, содержащую $O$ и ортогональную $\mathcal{H}_{\delta_{1}}$ и $\mathcal{H}_{\delta_{2}}$.

При использовании формулы (1.1.16) и “углов" $\theta\left(\delta_{1}\right), \theta\left(\delta_{2}\right), \theta\left(\delta_{12}\right)$ вместо углов $\theta_{1}, \theta_{2}, \theta_{12}$ в формуле (1.1.15) доказательство теоремы 1.1.3' аналогично доказательству теоремы 1.1.1' (см. доказательство теоремы 1 в [19, приложение]).

Докажем последнее утверждение. Пусть $K$ - фундаментальный многогранник для действия $A(\mathcal{M})$ на орисфере $\mathcal{E}_{O}$. Пусть $C_{K}$ - конус с вершиной $O$ и основанием $K$. Тогда $C_{K} \cap \mathcal{M}(W)$ является фундаментальным многогранником для полупрямого произведения $W . A(\mathcal{M})$, которое имеет конечный индекс в $O_{+}(S)$. Следовательно, $C_{K} \cap \mathcal{M}(W)$ - эллиптический многогранник. Отсюда следует, что $\mathcal{M}(W)$ - параболический многогранник относительно $O$. Множество гиперплоскостей $\mathcal{H}_{\delta}, \delta \in P(\mathcal{M})$, граней многогранника $\mathcal{M}(W)$, являюшихся одновременно гиперплоскостями граней многогранника $C_{K} \cap \mathcal{M}(W)$, конечно. Отсюда следует, что $r(\mathcal{M}(W))$ конечно и $\mathcal{M}(W)$ является ограниченным параболическим. Это завершает доказательство теоремы 1.1.3'.

Теперь теорема 1.1.3 следует из теоремы 1.1.3' так же, как теорема 1.1.1 следует из теоремы 1.1.1' (см. доказательство теоремы 5.2.1 в [20]). В [20] это сделано над произвольным подходяшим полем. Над $\mathbb{Q}$ доказательство очень простое.

Это завершает доказательство теоремы 1.1.3.

Э.Б. Винберг в [32] показал, что для эллиптических гиперболических рефлективных решеток $S$ ранг $\operatorname{rk} S \leqslant 30$. $\Phi$. Эссельман [8] улучшил этот результат (используя тот же метод) и показал, что $\mathrm{rk} S \leqslant 22$. Эта оценка для эллиптических рефлективных решеток является точной. Р. Борчердс [1] показал, что максимальная четная подрешетка нечетной унимодулярной гиперболической решетки ранга 22 эллиптическая рефлективная. Используя метод Винберга, можно показать, что ранг параболических рефлективных решеток $S$ также абсолютно ограничен. Например, нетрудно показать, что $\mathrm{rk} S \leqslant 43$ для параболических реффлективных решеток $S$. Здесь мы используем существование решетки Лича и двух четных унимодулярных решеток ранга 16 . Но можно ожидать, что точная оценка здесь должна быть $\operatorname{rk} S \leqslant 26$. Дж. Конвей [7] показал, что четная унимодулярная гиперболическая решетка ранга 26 является параболической рефилективной.

Отметим, что обшие результаты по ограничению размерности произвольных (т.е. не обязательно арифметических) групп отражений так называемых параболического и гиперболического типов в пространствах Лобачевского получены 
в [23] (см. также [24]). Они обобшают известные результаты автора [20], Э.Б. Винберга [32], М.Н. Прохорова [27] и А.Г. Хованского [14] по ограничению размерности эллиптических (т.е. с фундаментальным многогранником конечного объема) групп отражений в пространствах Лобачевского.

1.2. Скручивающие коэффициенты. Для алгебр Каца-Муди и обобщенных алгебр Каца-Муди, которые мы будем рассматривать ниже, гиперболическая решетка $S: M \times M \rightarrow \mathbb{Z}$ с точностью до подобия $S \mapsto S(m), m \in \mathbb{Q}$, является инвариантом алгебры или соответствующей обобщенной матрицы Картана. (Мы будем рассматривать только неразложимые обобщенные матрицы Картана.) Таким образом, естественно нормализовать $S$ условием, что она является примитивной или четной примитивной.

Напомним, что решетка $S$ является четной, если $S(x, x)$ четно для любого $x \in M$. Иначе решетка $S$ называется нечетной. Решетка $S$ называется примитивной (соответственно четной примитивной), если $S(1 / m)$ не является решеткой (соответственно четной решеткой) для любого натурального $m \in \mathbb{N}, m \geqslant 2$. Эти две нормализации отличаются тем, что если $S$ примитивна и нечетна, то $S(2)$ примитивна четна. Для большинства результатов, рассматриваемых ниже, не важно, какая из этих двух нормализаций выбирается. Таким образом, "примитивная" (решетка) означает примитивная или четная примитивная, если не сказано точно, какая из двух нормализаций выбирается.

Пусть $S: M \times M \rightarrow \mathbb{Z}$ - примитивная гиперболическая решетка. Мы фиксируем группу отражений $W \subset W(S)$ и фундаментальный многогранник $\mathcal{M}$ группы $W$. Пусть $P(\mathcal{M})_{\mathrm{pr}}$ - множество примитивных элементов решетки $M$, ортогональных $\mathcal{M}$. Пусть $A(\mathcal{M}) \subset O_{+}(S)$ - группа симметрий $\mathcal{M}$ (см. (1.1.10)).

ОПреДЕлениЕ 1.2.1. Функция $\lambda: P(\mathcal{M})_{\mathrm{pr}} \rightarrow \mathbb{N}$ называется функцией скручивающих коэффициентов, если

$$
S(\lambda(\delta) \delta, \lambda(\delta) \delta) \mid 2 S(M, \lambda(\delta) \delta) \quad \text { для любого } \delta \in P(\mathcal{M})_{\mathrm{pr}}
$$

и подгруппа

$$
A(P(\mathcal{M}))=\left\{g \in A(\mathcal{M}) \mid \lambda(g(\delta))=\lambda(\delta) \quad \text { для любого } \delta \in P(\mathcal{M})_{\mathrm{pr}}\right\}
$$

имеет конечный индекс в $A(\mathcal{M})$. Число $\lambda(\delta)$ назьвается скручивающим коэфффиииентом элемента $\delta \in P(\mathcal{M})_{\mathrm{pr}}$.

Очевидно, мы можем переформулировать это определение следующим образом. Рассмотрим новое множество $P(\mathcal{M})$ ортогональных векторов к $\mathcal{M}$ :

$$
P(\mathcal{M})=\left\{\alpha=\lambda(\delta) \delta \mid \delta \in P(\mathcal{M})_{\mathrm{pr}}\right\} .
$$

Это множество $P(\mathcal{M}) \subset M$ называется допустимым, если

$$
S(\alpha, \alpha) \mid 2 S(M, \alpha) \quad \text { для любого } \alpha \in P(\mathcal{M})
$$

и подгруппа

$$
A(P(\mathcal{M}))=\{g \in A(\mathcal{M}) \mid g(P(\mathcal{M}))=P(\mathcal{M})\}
$$


имеет конечный индекс в $A(\mathcal{M})$. Очевидно, (1.2.1) и $\left(1.2 .1^{\prime}\right)$ эквивалентны $(1.2 .2)$ и $\left(1.2 .2^{\prime}\right)$. Таким образом, допустимое множество $P(\mathcal{M})$ эквивалентно функции скручивающих коэффициентов. Если $P(\mathcal{M})$ допустимо, то

$$
P(\mathcal{M}) / P(\mathcal{M})_{\mathrm{pr}}
$$

- функция скручиваюших коэффициентов.

Нам понадобятся оценки $S(\delta, \delta), \lambda(\delta)$ для $\delta \in P(\mathcal{M})_{\operatorname{pr}}$ и $S(\alpha, \alpha)$ для $\alpha \in P(\mathcal{M})$, где $P(\mathcal{M})$ допустимо.

ПРЕДЛОЖЕНИЕ 1.2.2. Пусть $а(S)$ - показатель дискриминантной группы $A_{S}=M^{*} / M$. Пусть $\delta \in P(\mathcal{M})_{\mathrm{pr}}$ и а $(\delta)$ - такое наибольшее натуральное число, для которого $\delta / a(\delta) \in M^{*}$. Тогда $a(\delta) \mid a(S)$ и $\lambda(\delta)$ удовлетворяет (1.2.1), если и только если $\lambda(\delta) S(\delta, \delta) \mid 2 a(\delta)$. В частности, $\lambda(\delta) S(\delta, \delta) \mid 2 a(S)$, и для допустимого $P(\mathcal{M})$ и $\alpha \in P(\mathcal{M})$ имеем $S(\alpha, \alpha) \mid 4 a(S)^{2}$.

ДОКАЗАТЕЛЬСТво очевидно.

ЗАмечаниЕ 1.2 .3 . Пусть $P(\mathcal{M})$ - допустимое множество, рассмотренное выше. Обозначим

$$
M_{P(\mathcal{M})}^{*}=\left\{x \in M^{*}|S(\alpha, \alpha)| 2 S(x, \alpha) \quad \text { для любого } \alpha \in P(\mathcal{M})\right\} .
$$

Рассмотрим элементы $h_{j} \in M_{P(\mathcal{M})}^{*} \cap \mathbb{R}_{++} \mathcal{M}, j \in J$, где $J$ - счетное множество. Тогда матрица Грама элементов $P(\mathcal{M}) \cup\left\{h_{j} \mid j \in J\right\}$ определяет обобщенную алгебру Каца-Муди (см. [2]) с множеством простых вешественных корней $P(\mathcal{M})$, множеством простых мнимых корней $h_{j} \in M_{P(\mathcal{M})}^{*}, j \in J$, и группой Вейля $W$. Решетку $M$ (соответственно $M_{P(\mathcal{M})}^{*}$ ) следует рассматривать как "расширенную корневую подрешетку, порожденную вешественными простыми корнями" (соответственно "расширенную решетку корней", порожденную вешественными и мнимыми простыми корнями) по модулю ядра канонической симметрической билинейной формы. Таким образом, предложение 1.2.2 описьвает все возможности для той части этой матрищы Грама, которая связана с простыми вешественными корнями.

1.3. Эллиптические и параболические группы отражений с решеточным вектором Вейля. Мы фиксируем примитивную четную эллиптическую или параболическую решетку $S: M \times M \rightarrow \mathbb{Z}$ и эллиптическую или параболическую групп отражений $W \subset W(S)$. Пусть $\mathcal{M}$ - фундаментальный многогранник для $W$ и $P(\mathcal{M})$ - допустимое множество ортогональных векторов к $\mathcal{M}$. Для краткости мы называем пару $(S, P(\mathcal{M}))$ әллиптической или параболической соответственно.

ОПРЕДЕЛЕнИЕ 1.3.1. Элемент $\rho \in M \otimes \mathbb{Q}$ называется решеточны.м вектором Вейля (для) $P(\mathcal{M})$, если

$$
S(\rho, \alpha)=-S(\alpha, \alpha) / 2 \quad \text { для любого } \alpha \in P(\mathcal{M}) .
$$

Очевидно, вектор Вейля $\rho \in[P(\mathcal{M})]^{*} \subset M \otimes \mathbb{Q}$, если $\rho$ сушествует. Очевидно, $\mathbb{R}_{++} \rho \in \mathcal{M}$ и $\rho$ инвариантен относительно группы $A(P(\mathcal{M}))$. Отсюда следует, что 
$S(\rho, \rho)<0$, если $W$ эллиптична, и $\rho \in \mathbb{Q}_{++} c$ и $S(\rho, \rho)=0$, если $W$ параболична и $c-$ ее касп. Так как $P(\mathcal{M})$ порождает $M \otimes \mathbb{Q}$ (по теоремам 1.1.1' и 1.1.3'), то вектор Вейля $\rho \in M \otimes \mathbb{Q}$, очевидно, единствен при фиксированном $P(\mathcal{M}) \subset M$.

Мы хотим показать, что множество эллиптических или параболических пар $(S, P(\mathcal{M}))$ с решеточным вектором Вейля в существенном конечно. Здесь пара $(S, P(\mathcal{M}))$ изоморфна паре $\left(S^{\prime}, P^{\prime}\left(\mathcal{M}^{\prime}\right)\right)$, если существует изометрия $\phi: S \rightarrow S^{\prime}$ решеток такая, что $\phi(P(\mathcal{M}))=P^{\prime}\left(\mathcal{M}^{\prime}\right)$.

TЕОРема 1.3.2. При $\mathrm{rk} S \geqslant 3$ множество әллиптических пар $(S, P(\mathcal{M}))$ c решеточныцм вектором Вейля конечно с точностью до изоморфизма. Здесь $S$ - примитивная четная әллиптическая рефлективная решетка, $W \subset W(S)$ - әллиптическая (т.е. конечного индекса в $O(S)$ ) группа отражений, $\mathcal{M}$ фундаментальный многогранник для $W$ и $P(\mathcal{M}) \subset M$ - допустимое множество ортогональных векторов $к \mathcal{M}$.

ДокАЗАТЕльСТво. Мы фиксируем четную эллиптическую реф̆лективную решетку $S: M \times M \rightarrow \mathbb{Z}$ ранга $\operatorname{rk} S=n+1 \geqslant 3$ (мы уже знаем, что их множество конечно). Пусть $(S, P(\mathcal{M}))$ - допустимая пара с решеточным вектором Вейля.

По теореме $1.1 .1^{\prime}$ и предложению 1.2 .2 сушествуют такие элементы $\alpha_{1}, \ldots$ $\ldots, \alpha_{n+1} \in P(\mathcal{M})$, что они порождают подрешетку $M^{\prime} \subset M$ конечного индекса и матрица Грама этих элементов имеет ограниченные целые коэффициенты. Таким образом, существует только конечное число возможностей для таких матриц Грама. Зафиксируем одну из этих возможностей для матрицы Грама $A=\left(S\left(\alpha_{i}, \alpha_{j}\right)\right), \quad 1 \leqslant i, j \leqslant n+1$. Мы имеем $M^{\prime} \subset M \subset\left(M^{\prime}\right)^{*}$, где вложение $M^{\prime} \subset\left(M^{\prime}\right)^{*}$ задается матрицей Грама $A$. Следовательно, сушествует только конечное число возможностей для промежуточной решетки $M$. Зафиксируем одну из этих возможностей для $M$. Так как решетка $S$ невырождена, сушествует единственньй элемент $\rho \in M \otimes \mathbb{Q}$, удовлетворяющий определению 1.3 .1 для подмножества $\left\{\alpha_{1}, \ldots, \alpha_{n+1}\right\} \subset P(\mathcal{M})$.

По предложению 1.2 .2 квадраты $0<S(\alpha, \alpha) \leqslant 4 a(S)^{2}$ ограничены для $\alpha \in$ $P(\mathcal{M})$. Отсюда следует, что множество

$$
\left\{\alpha \in M \mid 0>S(\rho, \alpha)=-S(\alpha, \alpha) / 2 \geqslant-2 a(S)^{2}\right\}
$$

конечно. Поэтому существует только конечное число возможностей для подмножества $P(\mathcal{M}) \subset M$. Это доказьвает теорему.

Для параболического случая утверждение конечности будет следующим.

Теорема 1.3.3. Для каждой параболической пары $(S, P(\mathcal{M}))$ с решеточньцм вектором Вейля $\rho$ группа $A(P(\mathcal{M})) \subset O(S)_{\rho}=\{\phi \in O(S) \mid \phi(\rho)=\rho\}$ имеет конечный индекс в евклидовой кристаллографической группе $O(S)_{\rho}$.

Зафиксируем константу $D>0$. Тогда для ранга $\mathrm{rk} S \geqslant 3$ множество параболических пар $(S, P(\mathcal{M}))$ с решеточным вектором Вейля $\rho$ конечно $c$ точностью до изоморфизма, если индекс $\left[O(S)_{\rho}: A(P(\mathcal{M}))\right]<D$. Здесь $S-$ примитивная четная параболическая рефлективная решетка, $W \subset W(S)$ параболическая группа отражсений с каспом $\mathbb{R}_{++} c=\mathbb{R}_{++} \rho, \mathcal{M}-$ фундаментальный многогранник для $W$ и $P(\mathcal{M}) \subset M$ - допустимое множество ортогональных векторов к $\mathcal{M}$. 
ДоказАТЕЛЬСтво. Докажем первое утверждение. Пусть $(S, P(\mathcal{M}))$ - параболическая пара, соответствующая параболической группе отражений $W$ с фундаментальным многогранником $\mathcal{M}$ и допустимым множеством векторов $P(\mathcal{M})$, ортогональных $\mathcal{M}$, и с решеточным вектором Вейля $\rho \in \mathbb{Q}_{++} c$, где $c-$ касп для $W$.

Так как $\rho \in \mathbb{Q}_{++} c$, имеем $A(P(\mathcal{M})) \subset O(S)_{c}=O(S)_{\rho}$ и $A(P(\mathcal{M}))$ имеет конечньй индекс в $O(S) / W$. Пусть $K$ - фундаментальная область для группы $A(P(\mathcal{M}))$ на орисфере $\mathcal{E}_{O}$, где $O=\mathbb{R}_{++} c$, и $C_{K}-$ конус с вершиной $O$ и основанием $K$. Тогда $C_{K} \cap \mathcal{M}$ - фундаментальная область конечного объема для группы $W . A(P(\mathcal{M}))$ в $\mathcal{L}(S)$. Так как $\rho \in \mathbb{Q}_{++} c$ - вектор Вейля, очевидно, что $S(c, \alpha)<0$ для всех $\alpha \in P(\mathcal{M})$. Таким образом, никакая грань многогранника $\mathcal{M}$ не содержит касп $O$, и в то же время $O$ принадлежит $\mathcal{M}$. Отсюда следует, что фундаментальная область $K$ на орисфере $\mathcal{E}_{O}$ имеет конечный объем. Так как $O(S)_{\text {c }}$ дискретна на $\mathcal{E}_{O}$, группа $A(P(\mathcal{M}))$ имеет конечный индекс в $O(S)_{c}$. Это доказывает первое утверждение.

Докажем второе утверждение. Мы рассуждаем так же, как при доказательстве теоремы 1.3.2 (используя теоремы 1.1.3 и 1.1.3' вместо теорем 1.1.1 и 1.1.1'). Но в конце доказательства нужно использовать то, что число подгрупп $A \subset O(S)_{\rho}$ конечно для ограниченного индекса $\left[O(S)_{\rho}: A\right]<D$, и заменить множество (1.3.2) на множество

$$
\left\{\alpha \in M \mid 0>S(\rho, \alpha)=-S(\alpha, \alpha) / 2 \geqslant-2 a(S)^{2} \text { и } \mathcal{H}_{\alpha} \cap C_{K} \neq \varnothing\right\} .
$$

Здесь (как и вьше) $K$ - фундаментальная область для $A(P(\mathcal{M}))$ на орисфере $\mathcal{E}_{O}$, $O=\mathbb{R}_{++} \rho$, и $\mathcal{H}_{\alpha}-$ гиперплоскость, ортогональная $\alpha \in M$. Это завершает доказательство.

Следуюший пример показывает, что условие на индекс $\left[O(S)_{\rho}: A(P(\mathcal{M}))\right]<$ $D$ сушественно в теореме 1.3.3.

ПРИмеР 1.3.4. Рассмотрим четную примитивную гиперболическую решетку $M=\mathbb{Z} \delta_{1} \oplus \mathbb{Z} \delta_{2} \oplus \mathbb{Z} \delta_{3}$ ранга 3 , где

$$
S\left(\delta_{i}, \delta_{j}\right)=\left(\begin{array}{rrr}
2 & -2 & -2 \\
-2 & 2 & -2 \\
-2 & -2 & 2
\end{array}\right) .
$$

Здесь $\left\{\delta_{1}, \delta_{2}, \delta_{3}\right\}=P(\Delta)_{\mathrm{pr}}$, где $\Delta$ - фундаментальньй треугольник с нулевыми углами (т.е. с тремя вершинами на бесконечности) для группы $W^{(2)}(S)$, порожденной отражениями во всех элементах $\delta \in M$ таких, что $S(\delta, \delta)=2$. Это один из примеров 2-рефлективных решеток ранга 3 , которые были описаны в [18].

Пусть $O=\mathcal{H}_{\delta_{2}} \cap \mathcal{H}_{\delta_{3}}$. Здесь $O=\mathbb{R}_{++} c$, где $c=\delta_{2}+\delta_{3} \in M$. Мы имеем $c^{2}=0$, $S\left(c, \delta_{2}\right)=S\left(c, \delta_{3}\right)=0$ и $S\left(c, \delta_{1}\right)=-4$.

Обозначим

$$
\rho=c / 4, \quad e_{0}=\delta_{1}, \quad f_{01}=2 s_{\delta_{1}}\left(\delta_{2}\right), \quad f_{02}=2 s_{\delta_{1}}\left(\delta_{3}\right) .
$$

Легко проверяется, что

$$
S(\alpha, \alpha) \mid 2 S(M, \alpha), \quad S(\rho, \alpha)=-S(\alpha, \alpha) / 2
$$


для $\alpha=e_{0}, f_{01}, f_{02}$. Здесь $S\left(e_{0}, e_{0}\right)=2$ и $S\left(f_{01}, f_{01}\right)=S\left(f_{02}, f_{02}\right)=8$.

Рассмотрим такой $\phi \in O(S)_{\rho}$, что

$$
\phi\left(\delta_{2}\right)=\delta_{3}, \quad \phi\left(\delta_{3}\right)=s_{\delta_{3}}\left(\delta_{2}\right), \quad \phi\left(\delta_{1}\right)=s_{\delta_{3}}\left(\delta_{1}\right) .
$$

На орисфере $\mathcal{E}_{0}$ автоморфизм $\phi$ - это параллельный перенос, переводящий прямую $\mathcal{H}_{\delta_{2}}$ в прямую $\mathcal{H}_{\delta_{3}}$ и треугольник $\Delta$ в треугольник $\phi(\Delta)$ с общей вершиной $O$ и обшей стороной $\mathcal{H}_{\delta_{3}}$ с треугольником $\Delta$.

Для $k \in \mathbb{N}$ следуюшим образом определим фундаментальный многоугольник $\mathcal{M}_{k}$ для подгрупшы отражений $W_{k} \subset W^{(2)}(S)$ с допустимым множеством векторов $P\left(\mathcal{M}_{k}\right)$ :

$$
P\left(\mathcal{M}_{k}\right)=\left\{\phi^{t}\left(e_{0}\right) \mid t \in \mathbb{Z} \text { и } t \not \equiv 0 \quad \bmod k\right\} \cup\left\{\phi^{t}\left(f_{01}\right), \phi^{t}\left(f_{02}\right) \mid t \equiv 0 \bmod k\right\} .
$$

Очевидно, $P\left(\mathcal{M}_{k}\right)$ - это бесконечный многоугольник с нулевыми углами. Отсюда следует, что $\mathcal{M}_{k}$ - фундаментальный многоугольник для подгруппы $W_{k} \subset$ $W^{(2)}(S)$, порожденной отражениями во всех элементах $P\left(\mathcal{M}_{k}\right)$. По нашей конструкции $\phi^{k} \in A\left(P\left(\mathcal{M}_{k}\right)\right)$ порождает подгруппу конечного индекса в $O_{\rho}(S)$. В силу (1.3.4) множество $P\left(\mathcal{M}_{k}\right)$ допустимо и $\rho$-решеточный вектор Вейля для $P\left(\mathcal{M}_{k}\right)$. Отсюда следует, что $W_{k}$ - параболическая группа отражений с каспом $c$.

Очевидно, всепары $\left(S, P\left(\mathcal{M}_{k}\right)\right)$ различны, так как элементы $\alpha \in P\left(\mathcal{M}_{k}\right)$ имеют квадраты $S(\alpha, \alpha)=2$ или $S(\alpha, \alpha)=8$ и в точности $k-1$ последовательная сторона многоугольника $\mathcal{M}_{k}$ имеет ортогональные векторы из $P\left(\mathcal{M}_{k}\right)$ с квадратом 2 .

1.4. Группы отражений арифметического типа. Мы рассматриваем гиперболические решетки $S: M \times M \rightarrow \mathbb{Z}$ и группы отражений $W \subset W(S)$. Пусть $\mathcal{M}$ - фундаментальный многогранник для $W$ и $P(\mathcal{M})$ - допустимое множество векторов, ортогональных $\mathcal{M}$. Мы хотим определить класс таких групп, который интересен с точки зрения алгебр Каца-Муди. С точки зрения соответствующих алгебр Каца-Муди следующее определение означает, что мнимые корни "ведут себя хорошо" (см. п. 2.2).

ОПРЕДЕЛЕНИЕ 1.4.1. Рассмотрим целочисленньй конус (полугруппу)

$$
Q_{+}=\sum_{\alpha \in P(\mathcal{M})} \mathbb{Z}_{+} \alpha \subset M
$$

и соответствующий двойственный целочисленный конус

$$
Q_{+}{ }^{*}=\{x \in M \mid S(x, P(\mathcal{M})) \leqslant 0\} \subset M .
$$

Группа $W$ имеет арифметический тип, если

$$
V^{+}(S) \cap(M \otimes \mathbb{Q}) \subset \mathbb{Q}_{+} Q_{+} .
$$

Другими словами, это означает, что для любого $x \in M$ с квадратом $S(x, x)<0$ существуют $n \in \mathbb{N}$ и $a(\delta) \in \mathbb{Z}_{+}, \delta \in P(\mathcal{M})$, которые почти все равны 0 (т.е. только конечное число не равно нулю), такие, что

$$
n x= \pm \sum_{\alpha \in P(\mathcal{M})} a(\alpha) \alpha .
$$


Так как конус $\overline{V^{+}(S)}$ самодвойствен, т.е. ${\overline{V^{+}(S)}}^{*}=\overline{V^{+}(S)}$, группа отражений $W \subset W(S)$ имеет арифметический тип, если и только если

$$
Q_{+}{ }^{*}=\{x \in M \mid S(x, P(\mathcal{M})) \leqslant 0\} \subset M \cap \overline{V^{+}(S)} .
$$

Так как фундаментальный многогранник

$$
\mathcal{M}=\left(\mathbb{R}_{++} Q_{+}{ }^{*} \cap V^{+}(S)\right) / \mathbb{R}_{++} \subset \mathcal{L}(S)
$$

для $W$ локально конечно порожден и $M \otimes \mathbb{Q}$ всюду плотно в $M \otimes \mathbb{R}$, мы можем переформулировать это определение, используя соответствующие вешественные конусы.

Рассмотрим конус

$$
\mathbb{R}_{+} Q_{+}=\overline{\sum_{\alpha \in P(\mathcal{M})} \mathbb{R}_{+} \alpha} \subset M \otimes \mathbb{R}
$$

и соответствуюший двойственный конус

$$
\left(\mathbb{R}_{+} Q_{+}\right)^{*}=\{x \in M \otimes \mathbb{R} \mid S(x, P(\mathcal{M})) \leqslant 0\} .
$$

Тогда $W \subset W(S)$ имеет арифметический тип, если и только если

$$
\overline{V^{+}(S)} \subset \mathbb{R}_{+} Q_{+},
$$

эквивалентно

$$
\left(\mathbb{R}_{+} Q_{+}\right)^{*} \subset \overline{V^{+}(S)} .
$$

Таким образом, (1.4.3)-(1.4.5), (1.4.9), (1.4.10) - это эквивалентные определения того, что $W$ имеет арифметический тип. Очевидно, это определение не зависит от выбора допустимого множества $P(\mathcal{M})$ векторов, ортогональных $\mathcal{M}$.

Следует рассматривать "арифметический тип" как очень сильно ослабленное условие конечности объема фундаментального многогранника групш отражений. Другое объяснение важности этого типа групп состоит в том, что группы отражений арифметического типа максимальны: не существует группы отражений $W^{\prime} \subset W(S)$ с фундаментальным многогранником $\mathcal{M}^{\prime}$ таких, что $W \subset W^{\prime}$, $P(\mathcal{M}) \subset P\left(\mathcal{M}^{\prime}\right)$ и $W \neq W^{\prime}$ (эквивалентно $P(\mathcal{M}) \neq P\left(\mathcal{M}^{\prime}\right)$ ). Это следует из (1.4.5).

Хотя определение 1.4.1 очень естественно, оно оказывается слишком обшим (приводит в обшем случае к слишком “диким" группам). Поэтому мы определяем более узкий класс групп отражений $W$, которые приводят к "более хорошим автоморфным свойствам" формулы для знаменателя соответствующих алгебр Каца-Муди (см. §2). 
ОПРЕДЕЛЕНИЕ 1.4.2. Группа отражений $W$ имеет ограниченный арифметический тun, если $W$ имеет по крайней мере одно отражение и для допустимого множества $P(\mathcal{M})$ векторов, ортогональных $\mathcal{M}$, группа симметрий $A(P(\mathcal{M}))$ имеет конечньй индекс в $O(S) / W(S)$ (более точно это означает, что полупрямое произведение $W \cdot A(P(\mathcal{M}))$ имеет конечньй индекс в $O(S))$.

Имеет место следуюший результат, который показывает, что ограниченный арифметический тип влечет арифметический тип, и дает много примеров групп отражений $W$ ограниченного арифметического типа (и, следовательно, арифметического типа).

Tеорема 1.4.3. Пусть $S$ - гиперболическая решетка, $W \subset W(S)$ - группа отражений, содержащая по крайней мере одно отражение, $\mathcal{M}$ - фундаментальный многогранник для $W$ и $P(\mathcal{M})$ - допустимое множество ортогональных векторов $к \mathcal{M}$ (например, можно взять $\left.P(\mathcal{M})_{\mathrm{pr}}\right)$. Предположим, что группа симметрий $A(P(\mathcal{M}))$ имеет конечный индекс в $O(S) / W$ (т.е. $W . A(P(\mathcal{M}))$ имеет конечный индекс в $O(S))$. Другими словами, $W$ имеет ограниченный арифметический тип.

Тогда $W$ имеет арифметический тип (т.е. имеют место эквивалентные свойства (1.4.3)-(1.4.5), (1.4.9), (1.4.10)).

ДокАЗАТЕЛЬСТво. Предположим, что $W$ не имеет арифметический тип. Тогда (1.4.10) не верно. Поэтому сушествует такой элемент $q \in \mathbb{R}_{+} Q_{+}^{*}$, что $S(q, q)>0$. Мы имеем $\mathbb{R}_{+} \mathcal{M} \subset \mathbb{R}_{+} Q_{+}^{*}$, где $\mathcal{M}$ - фундаментальный многогранник для $W$ в $\mathcal{L}(S)$, которьй локально конечен в $\mathcal{L}(S)$ и невырожден. Здесь $\mathbb{R}_{+} \mathcal{M}=\left(\mathbb{R}_{+} Q_{+}^{*}\right) \cap \overline{V^{+}(S)}$. Рассматривая пересечение выпуклой оболочки $\mathbb{R}_{+} \mathcal{M}$ и $\mathbb{R}_{+} q$ с $V^{+}(S)_{\infty}$, легко видеть, что сушествует непустое открытое в $\overline{\mathcal{L}(S)}$ подмножество $U \subset \mathcal{M}$ такое, что $U \cap \mathcal{L}(S)_{\infty}$ является непустым открытым подмножеством в $\mathcal{L}(S)_{\infty}$. В частности, для всех $\alpha \in P(\mathcal{M})$ пересечение $\mathcal{H}_{\alpha} \cap U=\varnothing$.

Так как $W . A(P(\mathcal{M}))$ имеет конечньй индекс в $O(S)$, а фундаментальная область $\mathcal{D}$ для действия $A(P(\mathcal{M}))$ в $\mathcal{M}$ является фундаментальной областью для $W \cdot A(P(\mathcal{M}))$ в $\mathcal{L}(S)$, то $\mathcal{D}$ имеет конечный объем. Отсюда следует, что $\mathcal{D}$ - выпуклый многогранник в $\mathcal{L}(S)$, который является выпуклой оболочкой конечного множества точек в $\mathcal{L}(S)$ и на бесконечности $\mathcal{L}(S)$. Так как $W$ имеет по крайней мере одно отражение, сушествует грань коразмерности 1 многогранника $\mathcal{D}$, которая является пересечением $\mathcal{D}$ с гиперплоскостью $\mathcal{H}_{\alpha}, \alpha \in P(\mathcal{M})$. Рассмотрим два случая.

Предположим, что $\mathcal{D}$ ограничен. Легко видеть, что тогда найдется такой $g \in A(P(\mathcal{M}))$, что $g(\mathcal{D}) \subset U$. Тогда $\mathcal{H}_{g(\alpha)} \cap U$ непусто, где $g(\alpha) \in P(\mathcal{M})$. Противоречие.

Теперь предположим, что $\mathcal{D}$ имеет бесконечную вершину. Хорошо известно, что эта вершина есть $\mathbb{R}_{++} c$, где $0 \neq c \in M \cap \overline{V^{+}(S)}$ и $S(c, c)=0$. Кроме того, известно, что бесконечно удаленные точки $\mathbb{R}_{++} g(c), g \in W \cdot A(\mathcal{M})$, всюду плотны в $\mathcal{L}(S)_{\infty}$. В частности, найдется такой $g \in A(\mathcal{M})$, что $O=\mathbb{R}_{++} g(c) \in U \cap \mathcal{L}(S)_{\infty}$, потому что $U \subset \mathcal{M}$. Тогда $g(\mathcal{D})$ - также фундаментальная область для $A(P(\mathcal{M}))$ в $\mathcal{M}$. По нашей конструкции гиперплоскости граней коразмерности 1 для $g(\mathcal{D})$, содержащие $O=\mathbb{R}_{++} g(c)$, отличны от $\mathcal{H}_{\alpha}, \alpha \in P(\mathcal{M})$. Отсюда следует, что стационарная 
подгруппа $A(P(\mathcal{M}))_{g(c)}$ для $g(c)$ имеет конечный индекс в $O(S)_{g(c)}$ и имеет фундаментальную область конечного объема на орисфере $\mathcal{E}_{O}$. Тогда гиперплоскости $\mathcal{H}_{f(g(\alpha))}$, где $f \in A(P(\mathcal{M}))_{g(c)}$, всюду плотны в $U \cap \mathcal{L}(S)_{\infty}$. Противоречие. Это завершает доказательство.

ЗАмЕчАниЕ. Теорему 1.4.3 можно рассматривать как частный случай некоторых общих результатов о предельных множествах дискретных групп в пространствах Лобачевского и их номальных подгрупп; см. [9] и обзор [35].

Легко построить множество примеров груп отражений ограниченного арифметического типа и, следовательно, по теореме 1.4.3, арифметического типа. Следующая конструкция инспирирована изучением групп автоморфизмов поверхностей Энриквеса в [21], [22].

ОПРЕДЕЛЕНИЕ 1.4.4. Рассмотрим конечную симметрическую билинейную (или подходящую квадратичную форму) $f: A \times A \rightarrow \mathbb{Z} / d$, где $A$ - конечная абелева группа и $d \in \mathbb{N}$. Подмножество $\bar{\Delta} \subset A$ называется конечной системой корней $\boldsymbol{в}(f, A)$, если сушествует целочисленная решетка $T: N \times N \rightarrow \mathbb{Z}$, ее подрешетка $N_{1} \subset N$ конечного индекса, группа отражений $W \subset W(T)$ и отождествление

$$
\left(N / N_{1}, T \bmod N_{1}\right)=(A, f)
$$

такие, что

$$
\bar{\Delta}=\left\{\delta \bmod N_{1} \mid s_{\delta} \in W \quad \text { и } \delta \text { является примитивным в } N\right\} .
$$

Было бы интересно дать разумную классификацию конечных систем корней.

Рассмотрим теперь гиперболическую решетку $S: M \times M \rightarrow \mathbb{Z}$, ее подрешетку $M_{1} \subset M$ конечного индекса и конечную систему корней $\bar{\Delta}$ в $\left(S \bmod M_{1}, M / M_{1}\right)$. Пусть

$$
W(\bar{\Delta}) \subset W(S)
$$

- группа, порожденная всеми отражениями $s_{\delta}$, где $\delta \in M$ примитивен и $\delta$ $\bmod M_{1} \in \bar{\Delta}$.

Так как $\bar{\Delta}$ конечно, используя теорему 1.4 .3$, получаем

СЛЕДСТВИЕ 1.4.5. Группа отражений $W(\bar{\Delta})$ имеет ограниченный арифметический тип (и, следовательно, арифметический тип), если $W(\bar{\Delta})$ содержит по крайней мере одно отражсение.

По предложению 1.2.2 квадраты $S(\delta, \delta)$ отражений $s_{\delta}$ с примитевным $\delta$ ограничены. Поэтому, используя следствие 1.4.5, для подходящей конечной системы корней $\bar{\Delta}$ мы формально получаем следующее наиболее полезное в приложениях утверждение 1.4.6. С другой стороны, это утверждение следует из теоремы 1.4 .3 непосредственно. 
СЛЕДСТВИЕ 1.4.6. Пусть фиксировано подмножество $\left\{d_{1}, \ldots, d_{k}\right\} \subset \mathbb{N} u$ подгруппа $W \subset W(S)$ порождена всеми отражениями в примитивных әлементах $\delta \in M$ таких, что $S(\delta, \delta) \in\left\{d_{1}, \ldots, d_{k}\right\}$.

Тогда $W$ имеет ограниченный арифметический тип (u, следовательно, арифметический тип), если она содержит хотя бы одно отражение.

Для $S(\delta, \delta)=2$ это утверждение доказано в [15].

Следующее утверждение показывает, что всякая конечно порожденная группа отражений гиперболической решетки содержится в группе отражений ограниченного арифметического типа этой же решетки. Его интерпретацию для алгебр Каца-Муди см. в $\S 2$.

TеОРема 1.4.7. Пусть $S: M \times M \rightarrow \mathbb{Z}$ - әиперболическая решетка $и$ $W \subset W(S)$ - конечно порожденная группа отражений с фундаментальным многогранником $\mathcal{M}$ и конечным допустимым множеством $P(\mathcal{M})$ векторов, ортогональных $\mathcal{M}$.

Тогда $W$ является подгруппой $W \subset W^{\prime} \subset W(S)$ группь отражений $W^{\prime}$ ограниченного арифметического типа с фундаментальным многогранником $\mathcal{M}^{\prime} \subset \mathcal{M}$ и допустимым множеством $P\left(\mathcal{M}^{\prime}\right)$, содержащим $P(\mathcal{M})$.

ДокАЗАТЕЛЬСТво. Используя предложение 1.2.2, достаточно доказать теорему для примитивных векторов $P(\mathcal{M})_{\text {pr }}$ и $P\left(\mathcal{M}^{\prime}\right)_{\mathrm{pr}}$.

Для группы отражений $\widetilde{W} \subset W(S)$ обозначим через

$$
\Delta(\widetilde{W})=\left\{\text { примитивный } \delta \in M \mid s_{\delta} \in \widetilde{W}\right\}
$$

множество всех примитивных корней группы отражений $\widetilde{W}$.

Пусть $h \in M, \mathbb{R}_{++} h \in V^{+}(S)$ и $0 \notin S(h, \Delta(W))$. Имеется следуюший алгоритм Винберга [31] для вычисления фундаментального многогранника $\widetilde{\mathcal{M}}$ группы $\widetilde{W}$ (эквивалентно множества $P(\widetilde{\mathcal{M}})_{\text {pr }}$ ), содержащего $\mathbb{R}_{++} h$ (эквивалентно $\left.S\left(h, P(\widetilde{\mathcal{M}})_{\mathrm{pr}}\right)<0\right)$. Вводится понятие высоты

$$
\operatorname{height}(\delta)=-2 S(h, \delta) / \sqrt{S(\delta, \delta)}, \quad \delta \in \Delta(\widetilde{W}) .
$$

Геометрически высота эквивалентна расстоянию между точкой $\mathbb{R}_{++} h$ и гиперплоскостью $\mathcal{H}_{\delta}$ в пространстве Лобачевского $\mathcal{L}(S)$. Согласно [31]

$$
P(\widetilde{\mathcal{M}})_{\mathrm{pr}}=\bigcup_{n \in \mathbb{N}} P(\widetilde{\mathcal{M}})_{\mathrm{pr}}^{(n)}
$$

где

$$
\begin{aligned}
P(\widetilde{\mathcal{M}})_{\mathrm{pr}}^{(n)}=\{\delta \in \Delta(\widetilde{W}) \mid \operatorname{height}(\delta)=\sqrt{n} \\
\text { и } \left.S\left(\delta, P(\mathcal{M})_{\mathrm{pr}}^{(m)}\right) \leqslant 0 \quad \text { для всех } 1 \leqslant m<n\right\} .
\end{aligned}
$$

Верно также, что множество

$$
\{\delta \in \Delta(W(S)) \mid 0 \leqslant \operatorname{height}(\delta) \leqslant C\}
$$


конечно для фикикированного $C>0$.

Теперь докажем теорему. Мы фиксируем такой $h \in M$, что $\mathbb{R}_{++} h \in \mathcal{M}$ и $S\left(h, P(\mathcal{M})_{\text {pr }}\right)<0$ (т.е. $\mathbb{R}_{++} h$ лежит внутри $\left.\mathcal{M}\right)$. Мы используем этот элемент $h$ для определения высоты. Пусть $N$ - наибольшая высота элементов конечного множества $P(\mathcal{M})_{\mathrm{pr}}$.

Мы рассматриваем подрешетку $M_{1} \subset M$ конечного индекса и конечную симметрическую билинейную форму

$$
\left(S \bmod M_{1}, M / M_{1}\right)
$$

с конечной системой корней

$$
\overline{\Delta(W)}=\Delta(W) \quad \bmod M_{1}
$$

такие, что множество

$$
\left\{\delta \in \Delta(W(S)) \mid 0 \leqslant \operatorname{height}(\delta) \leqslant N \quad \text { и } \quad \delta \quad \bmod M_{1} \in \overline{\Delta(W)}\right\}
$$

равно множеству

$$
\{\delta \in \Delta(W) \mid 0 \leqslant \text { height }(\delta) \leqslant N\} .
$$

Так как множество (1.4.18) конечно, мы всегда можем удовлетворить этому равенству для достаточно глубокой подрешетки $M_{1} \subset M$. Применим алгоритм Винберга к вычислению $P(\mathcal{M})_{\text {pr }}$ и фундаментального многогранника $\mathcal{M}_{1}$ (эквивалентно $\left.P\left(\mathcal{M}_{1}\right)_{\text {pr }}\right)$ группы отражений $W(\overline{\Delta(W)})$. По вьшеприведенному условию равенства множеств мы имеем $P\left(\mathcal{M}_{1}\right)_{\mathrm{pr}}^{(n)}=P(\mathcal{M})_{\mathrm{pr}}^{(n)}$ для всех $0 \leqslant n \leqslant N$. Отсюда следует, что $P(\mathcal{M})_{\mathrm{pr}} \subset P\left(\mathcal{M}_{1}\right)_{\mathrm{pr}}$. По следствию 1.4 .5 группа отражений $W(\overline{\Delta(W)})$ имеет ограниченный арифметический тип, так как содержит нетривиальную подгруппу отражений $W$. Это завершает доказательство.

Используя ту же самую идею, можно доказать следующее более общее утверждение.

ТЕОРемА 1.4.8. Пусть $S: M \times M \rightarrow \mathbb{Z}$ - гиперболическая решетка и $W \subset W(S)$ - конечно порожденная группа отражсений с фундаментальным многогранником $\mathcal{M}$ и конечным допустимым множсеством $P(\mathcal{M})$ векторов, ортогональных $\mathcal{M}$. Пусть $h_{1}, \ldots, h_{k} \in(M-\{0\}) \cap \mathbb{R}_{++} \mathcal{M}$ (әквивалентно $h_{i} \in(M-\{0\}) \cap \overline{V^{+}(S)}$ u $\left.S\left(h_{i}, P(\mathcal{M})\right) \leqslant 0, \quad i=1, \ldots, k\right)$. Тогда $W$ является подгруппой отражсний $W \subset W^{\prime} \subset W(S)$ группы отражсений $W^{\prime}$ ограниченного арифметического типа с фундаментальным многогранником $\mathcal{M}^{\prime} \subset \mathcal{M} u$ допустимым множеством $P\left(\mathcal{M}^{\prime}\right)$, которое содержит $P(\mathcal{M})$. Кроме того, $h_{1}, \ldots, h_{k} \in(M-\{0\}) \cap \mathbb{R}_{++} \mathcal{M}^{\prime}$.

В конще этого параграфа мы рассмотрим связь между эллиптическими и параболическими группами отражений и группами отражений арифметического и ограниченного арифметического типа.

Имеет место следующее естественное обобщение определения 1.3.1. 
ОПРЕДЕЛЕНИЕ 1.4.9. Пусть $S: M \times M \rightarrow \mathbb{Z}$-гиперболическая решетка и $W \subset$ $W(S)$ - группа отражений с фундаментальным многогранником $\mathcal{M}$ и допустимым множеством $P(\mathcal{M})$ векторов, ортогональных $\mathcal{M}$.

Элемент $0 \neq \rho \in M$ назьвается обобщенным решеточным вектором Вейля для $P(\mathcal{M})$, если найдется такая константа $C>0$, что

$$
0 \leqslant-S(\rho, \alpha) \leqslant C \text { для любого } \alpha \in P(\mathcal{M})
$$

Элемент $\rho \in M \otimes \mathbb{Q}$ называется решеточны.м вектором Вейля для $P(\mathcal{M})$, если

$$
S(\rho, \alpha)=-S(\alpha, \alpha) / 2 \quad \text { для любого } \alpha \in P(\mathcal{M}) .
$$

Очевидно, что некоторая кратность решеточного вектора Вейля дает обобщенный решеточный вектор Вейля. Сушествование обобщенного решеточного вектора Вейля не зависит от выбора допустимого множества $P(\mathcal{M})$ векторов, ортогональных $\mathcal{M}$.

Мы имеем следуюшие утверждения, смысл которых в том, что эллиптические группы отражений - это в точности группы отражений арифметического типа с обобщенным решеточным вектором Вейля $\rho$, имеющим отрицательный квадрат $S(\rho, \rho)<0$, а параболические группы отражений - это в точности группы отражений ограниченного арифметического типа, имеющие обобщенный решеточный вектор Вейля $\rho$ с нулевым квадратом $S(\rho, \rho)=0$ и не имеюшие обобщенньй решеточный вектор Вейля с отрицательным квадратом.

ПРЕДЛОЖЕНИЕ 1.4.10. Любая әллиптическая или параболическая группа отражений $W \subset W(S)$ гиперболической решетки $S$ имеет ограниченный арифметический тип (и по теореме 1.4 .3 имеет арифметический тип). Если $W$ әллиптична, то она имеет обобщенный решеточный вектор Вейля $\rho$ с отрицательным квадратом $S(\rho, \rho)<0$. Если $W$ параболична, она имеет обобщенный решеточный вектор Вейля $\rho$ с нулевым квадратом $S(\rho, \rho)=0$ u не имеет обобщенный решеточный вектор Вейля с отрицательным квадратом.

ДоКАЗАТЕЛЬСтво. Из определения сразу следует, что такая группа имеет ограниченный арифметический тип. По теореме 1.4.3 она имеет арифметический тип. Пусть $\mathcal{M}$ - фундаментальный многогранник для $W$. Если $W$ эллиптична, любой элемент $0 \neq \rho \in M$ такой, что $\mathbb{R}_{++} \rho \in \operatorname{Int} \mathcal{M}$ (эквивалентно $S(\rho, P(\mathcal{M}))<0$ ) является обобшенным решеточным вектором Вейля с $S(\rho, \rho)<0$. Если $W$ параболична, то касп $c$ является обобщенным решеточным вектором Вейля с $S(c, c)=0$. Ниже мы покажем, что $P(\mathcal{M})$ не имеет обобщенного решеточного вектора Вейля с отрицательным квадратом.

ПРЕДЛОЖЕНИЕ 1.4.11. Пусть $S$ - гиперболическая решетка и $W \subset W(S)$ группа отражений арифметического типа, $\mathcal{M}$ - фундаментальный многогранник для $W$ и $P(\mathcal{M})$ - допустимое множество векторов, ортогональных M. Предположим, что $P(\mathcal{M})$ имеет обобщенный решеточный вектор Вейля $\rho$. 
Тогда $S(\rho, \rho) \leqslant 0$. При этом если $S(\rho, \rho)<0$, то $W$ әллиптична; если $S(\rho, \rho)=0$ и $W$ имеет ограниченный арифметический тип, то $W$ либо эллиптична, либо параболична.

ДокАЗАТЕЛЬСтво. По определению обобщенного решеточного вектора Вейля $\rho$ имеем $S(\rho, P(\mathcal{M})) \leqslant 0$. Таким образом, $\rho \in \mathbb{R}_{+} Q_{+}{ }^{*}$ (см. определение 1.4.1). В силу (1.4.5) $\rho \in \overline{V^{+}(S)}$. Отсюда следует, что $S(\rho, \rho) \leqslant 0$ и $\mathbb{R}_{++} \rho \in \mathcal{M}$.

Предположим, что $S(\rho, \rho)<0$. По определению обобщенного решеточного вектора Вейля тогда $P(\mathcal{M})$ конечно (так как решетка $S$ гиперболична). В силу (1.4.6) и (1.4.10) фундаментальный многогранник $\mathcal{M}=\left(\mathbb{R}_{+} Q_{+}\right)^{*} / \mathbb{R}_{++}$является выпуклой оболочкой конечного множества точек из $\mathcal{L}(S)$ и $\mathcal{L}(S)_{\infty}$. Таким образом, $\mathcal{M}$ имеет конечный объем и $W$ эллиптична.

Теперь предположим, что $S(\rho, \rho)=0$ и $W$ имеет ограниченный арифметический тип. Если $A(P(\mathcal{M}))$ конечна, то $W \subset O(S)$ имеет конечный индекс и $W$ эллиптична. Предположим, что $A(P(\mathcal{M}))$ бесконечна. Очевидно, что $g(\rho)$ также является обобщенным решеточным вектором Вейля для любого $g \in A(P(\mathcal{M}))$. Если $g(\rho) \neq \rho$, отсюда следует, что $P(\mathcal{M})$ конечно. Так как $W$ имеет арифметический тип, рассуждая так же, как и выше, доказывается, что $W$ эллиптична и $A(P(\mathcal{M}))$ конечна. Получаем противоречие. Таким образом, мы показали, что $g(\rho)=\rho$ для любого $g \in A(P(\mathcal{M}))$. Тогда $\rho$ является каспом для $A(P(\mathcal{M}))$. Так как $W$ имеет ограниченный арифметический тип, $W \cdot A(P(\mathcal{M}))$ имеет конечный индекс в $O(S)$. Отсюда следует, что $W$ параболична.

ЗАмЕчАНИЕ 1.4.12. Модифицируя пример 1.3.4, легко построить пример группы отражений $W$ арифметического типа и с решеточным вектором Вейля $\rho$ с нулевым квадратом $S(\rho, \rho)=0$ такой, что данная группа не имеет ограниченный арифметический тип, и поэтому не является параболической.

\section{§2. Формула для знаменателя лоренцевых алгебр Каца-Муди}

Мы ограничимся рассмотрением алгебр Каца-Муди, но аналогично можно рассматривать обобщенные алгебры Каца-Муди (см. замечание 1.2.3 и теорему 1.4.8) и, по-видимому, подходящие обобщенные супералгебры Каца-Муди.

2.1. Основные определения, связанные с лоренцевыми алгебрами Каца-Муди. По поводу основных определений, связанных с алгебрами Каца-Муди, мы отсылаем к работам В. Каца [13] и Р. Борчердса [2].

Мы фиксируем четную примитивную гиперболическую решетку $S$ : $M \times M \rightarrow \mathbb{Z}$, конечно порожденную группу отражений $W \subset W(S)$, ее фундаментальный многогранник $\mathcal{M}$ и конечное допустимое множество $P(\mathcal{M})$ векторов, ортогональных $\mathcal{M}$. Мы предполагаем, что $P(\mathcal{M})$ порождает подрешетку конечного индекса в $M$ и граф Грама множества $P(\mathcal{M})$ связен. Последнее означает, что не существует разбиения $P(\mathcal{M})$ на два взаимно ортогональных непустых подмножества. Мы это предполагаем, чтобы не рассматривать конечномерные и аффинные алгебры Каца-Муди. 
Рассмотрим конечную матрищу

$$
A=\left(a_{i j}\right)=\left(\frac{2 S\left(\alpha_{i}, \alpha_{j}\right)}{S\left(\alpha_{i}, \alpha_{i}\right)}\right), \quad \alpha_{i}, \alpha_{j} \in P(\mathcal{M})
$$

Так как $\mathcal{M}$ является фундаментальным многогранником групшы отражений и $P(\mathcal{M})$ допустимо, то $a_{i i}=2, a_{i j}$ неположительные и целые для $i \neq j$ и $a_{i j}, a_{j i}$ равны нулю одновременно. Матрица $\left(a_{i j}\right)$ с этими свойствами называется обобщенной матрицей Картана. Обобщенная матрица Картана $\left(a_{i j}\right)$, определенная в (2.1.1), специального типа. Прежде всего, она симметризуема, что означает, что

$$
A=D B
$$

где $D$ диагональна с положительными рациональными коэффициентами и $B$ симметрическая. Очевидно, для нашего случая

$$
D=\operatorname{diag}\left(\ldots, 2 / S\left(\alpha_{i}, \alpha_{i}\right), \ldots\right)
$$

и

$$
B=\left(b_{i j}\right)=\left(S\left(\alpha_{i}, \alpha_{j}\right)\right)
$$

является матрицей Грама $P(\mathcal{M})$. Так как граф Грама, определяемый $B$, связен, матрица $A$ неразложима. Кроме того, для нашего случая матрица $B$ гиперболична, т.е. имеет ровно один отрицательный квадрат (и несколько положительных и нулевых квадратов).

Мы называем обобшенную матрищу Картана с вьшеперечисленными свойствами неразложимой лоренцевой симметризуемой. По вышеприведенной конструкции легко видеть, что любая неразложимая лоренцева симметризуемая обобщенная матрица Картана $A$ определяет примитивную четную гиперболическую решетку $S$, группу отражений $W \subset W(S)$ и множество $P(\mathcal{M})$ канонически с точностью до изоморфизма и конечного числа возможностей. Если потребовать, чтобы множество $P(\mathcal{M})$ порождало $M$, то $S, W, P(\mathcal{M})$ будут определены канонически с точностью до изоморфизма.

Пусть $P(\mathcal{M})=\left\{\alpha_{1}, \ldots, \alpha_{N}\right\}$. Обобщенная матрица Картана $A$, определенная вьше, задает алгебру Каца-Муди $\mathfrak{g}^{\prime}(A)$ над $\mathbb{C}$. Алгебра Ли $\mathfrak{g}^{\prime}(A)$ задается $3 N$ образуюшими $e_{1}, \ldots, e_{N}, f_{1}, \ldots, f_{N}, h_{1}, \ldots, h_{N}$ и определяюшими соотношениями

$$
\begin{aligned}
{\left[h_{i}, h_{j}\right]=0, \quad\left[e_{i}, f_{j}\right] } & =\delta_{i j} h_{j}, \quad\left[h_{i}, e_{j}\right]=a_{i j} e_{j}, \quad\left[h_{i}, f_{j}\right]=-a_{i j} f_{j} \\
\left(\operatorname{ad} e_{i}\right)^{1-a_{i j}} e_{j} & =0, \quad\left(\operatorname{ad} f_{i}\right)^{1-a_{i j}} f_{j}=0, \quad \text { если } i \neq j
\end{aligned}
$$

для всех $1 \leqslant i, j \leqslant N$.

Основное свойство этой алгебры Каца-Муди заключается в том, что

$$
\mathfrak{g}^{\prime \prime}(A)=\mathfrak{g}^{\prime}(A) / \mathfrak{t}=\bigoplus_{\alpha \in M} \mathfrak{g}_{\alpha}
$$

является простой и градуированной решеткой $M$. Здесь $\mathfrak{t}$ - центр алгебры $\mathfrak{g}^{\prime}(A)$; $\operatorname{dim} \mathfrak{g}_{\alpha}<\infty,\left[\mathfrak{g}_{\alpha}, \mathfrak{g}_{\beta}\right] \subset \mathfrak{g}_{\alpha+\beta} ; \mathfrak{g}_{0}=\mathbb{C} h_{1}+\cdots+\mathbb{C} h_{N}=M^{*} \otimes \mathbb{C}$, где $\left\langle h_{i}, \alpha_{j}\right\rangle=a_{i j} ;$ 
элемент $X \in \mathfrak{g}_{\alpha}$, если и только если $[h, X]=\langle h, \alpha\rangle X$ для любого $h \in \mathfrak{g}_{0}$. Форма $S$ может быть продолжена канонически на $\mathfrak{g}^{\prime \prime}(A)$ до инвариантной симметрической билинейной формы. Она называется канонической симметрической билинейной формой.

Часть $\mathfrak{g}_{0}$ называется подалгеброй Картана алгебры $\mathfrak{g}^{\prime \prime}(A)$. Элемент $0 \neq \alpha \in$ $M$ называется корнем, если кратность $\operatorname{mult}(\alpha)=\operatorname{dim} \mathfrak{g}_{\alpha}>0$. Пусть $\Delta-$ множество всех корней. Корни и их кратности инвариантны относительно $W$, которая назьвается группой Вейля.

Согласно [13] имеет место следуюшее описание множества корней $\Delta$. Корень $\alpha \in M$ называется вещественнылм, если $S(\alpha, \alpha)>0$. Корень $\alpha \in M$ называется мнимым, если $S(\alpha, \alpha) \leqslant 0$. Множество вещественных корней обозначается $\Delta^{\mathrm{re}}$, множество мнимых корней обозначается $\Delta^{\mathrm{im}}$. Пусть $Q_{+}=\mathbb{Z}_{+} \alpha_{1}+\cdots+\mathbb{Z}_{+} \alpha_{N}-$ целочисленный конус, порожденный простыми корнями (мы уже определяли и использовали этот конус в (1.4.1)). Имеем $\Delta=\Delta_{+} \cup \Delta_{-}$, где $\Delta_{+}=\Delta \cap Q_{+}$, $\Delta_{-}=-\Delta_{+}$. Аналогично определяются $\Delta_{+}^{\mathrm{re}}, \Delta_{-}^{\mathrm{re}}$ и $\Delta_{+}^{\mathrm{im}}, \Delta_{-}^{\mathrm{im}}$. Очевидно, что $\alpha \in \Delta_{+}$, если и только если $S(\alpha, h) \leqslant 0$ для любого $\mathbb{R}_{++} h \in \mathcal{M}$. Мнимый корень $\alpha \in \Delta_{+}$, если и только если $\alpha \in \overline{V^{+}(S)}$. Элементы $\left\{\alpha_{1}, \ldots, \alpha_{N}\right\}=P(\mathcal{M})$, очевидно, являются вещественными корнями. Они называются простыми вещественными корня.ми и имеют кратность один. Имеем

$$
\Delta^{\mathrm{re}}=W(P(\mathcal{M}))
$$

Пусть

$$
Q_{+}{ }^{*}=\{x \in M \mid S(x, P(\mathcal{M})) \leqslant 0\}
$$

- целочисленный конус, двойственный к $Q_{+}$, и

$$
K=Q_{+} \cap Q_{+}{ }^{*}
$$

Согласно [13] (см. также рассуждения в [25]) имеем

$$
\Delta_{+}^{\mathrm{im}}=W(K) .
$$

Рассмотрим соответствуюшие вешественные конусы

$$
\mathbb{R}_{+} Q_{+}, \quad\left(\mathbb{R}_{+} Q_{+}\right)^{*} \text { и } \mathbb{R}_{+} K=\left(\mathbb{R}_{+} Q_{+}\right) \cap\left(\mathbb{R}_{+} Q_{+}\right)^{*} .
$$

Мы имеем

$$
\mathbb{R}_{+} K \subset \mathbb{R}_{+} \mathcal{M}
$$

Конус

$$
T^{*}=W\left(\mathbb{R}_{+} K\right)=W\left(\mathbb{R}_{+} Q_{+} \cap\left(\mathbb{R}_{+} Q_{+}\right)^{*}\right) \subset \overline{V^{+}(S)}
$$

называется двойственным конусом Титса. Соответственно $T=\left(T^{*}\right)^{*}$ называется конусом Титса. Очевидно,

$$
\Delta_{+}^{\mathrm{im}}=Q_{+} \cap T^{*} .
$$


Имеет место следуюшая формула Вейля-Каца для знаменателя:

$$
\Phi(z)=: \sum_{w \in W} \operatorname{det}(w) e^{-2 \pi i S(w(\rho), z)}=e^{-2 \pi i S(\rho, z)} \prod_{\alpha \in \Delta_{+}}\left(1-e^{-2 \pi i S(\alpha, z)}\right)^{\operatorname{mult}(\alpha)} .
$$

Здесь $\rho \in\left(\oplus \mathbb{Z} \alpha_{i}\right)^{*}$ - любой элемент, удовлетворяюший равенству

$$
\left\langle\rho, \alpha_{i}\right\rangle=-S\left(\alpha_{i}, \alpha_{i}\right) / 2 \text { для любого } \alpha_{i} \in P(\mathcal{M}) \text {. }
$$

Он называется вектором Вейля. Переменная $z$ пробегает

$$
M \otimes \mathbb{C}
$$

Область $\Omega(S)=M \otimes \mathbb{R}+i V^{+}(S) \subset M \otimes \mathbb{C}$ называется комплексифицированным конусом $V^{+}(S)$. Известно [13], что $\Phi(z)$ абсолютно сходится в комплексифицированном конусе Титса

$$
\Omega(S, W)=M \otimes \mathbb{R}+i \operatorname{Int} T \subset S \otimes \mathbb{C},
$$

если $\operatorname{Im} z \gg 0$, и абсолютно расходится в $S \otimes \mathbb{C}-\overline{\Omega(S, W)}$. Таким образом, область $\Omega(S, W)$ сходимости $\Phi(z)$ всегда содержит естественную область $\Omega(S)$.

Мы обозначаем обобщенную матрицу Картана $A$ в $(2.1 .1)$ как $A(S, W, P(\mathcal{M}))$ и соответствуюшие алгебры Каца-Муди $\mathfrak{g}^{\prime}(A)$ и $\mathfrak{g}^{\prime \prime}(A)$ - как $\mathfrak{g}^{\prime}(A(S, W, P(\mathcal{M})))$ и $\mathfrak{g}^{\prime \prime}(A(S, W, P(\mathcal{M})))$. Они называются лоренцевыми алгебрами Каца-Муди.

Видно, что лоренцевы алгебры Каца-Муди $\mathfrak{g}^{\prime \prime}(A(S, W, P(\mathcal{M})))$ в общем случае имеют плохие свойства.

(-а) Рациональньй конус $\mathbb{Q}_{+} \Delta_{+}^{\mathrm{im}}$, порожденньй мнимыми корнями, дает только часть естественного конуса $(M \otimes \mathbb{Q}) \cap V^{+}(S)$.

(-b) Функция для знаменателя $\Phi(z)$ сходится в большей области $\Omega(S, W)$, чем естественная область $\Omega(S)$.

$(-c)$ Функция для знаменателя $\Phi(z)$ антиинвариантна относительно группы Вейля $W$ и инвариантна отностительно конечной группы $A(P(\mathcal{M}))$ симметрий $\mathcal{M}$. Cоответствуюшее полупрямое произведение $W . A(S)$ может быть очень мало в естественной арифметической группе $O(S)$.

$(-\mathrm{d})$ Выражения $S(\rho, z)$ и $S(w(\rho), z)$ не определены корректно, если не сушествует $\rho \in M \otimes \mathbb{Q}$ такого, что

$$
S\left(\rho, \alpha_{i}\right)=-S\left(\alpha_{i}, \alpha_{i}\right) / 2
$$

Если такой $\rho \in M \otimes \mathbb{Q}$ сушествует, то говорят, что решетка $S$ имеет решеточный вектор Вейля. Если решеточный вектор Вейля не существует, то следует переписать формулу (2.1.15) в виде

$$
\sum_{w \in W} \operatorname{det}(w) e^{-2 \pi i S(w(\rho)-\rho, z)}=\prod_{\alpha \in \Delta_{+}}\left(1-e^{-2 \pi i S(\alpha, z)}\right)^{\operatorname{mult}(\alpha)} .
$$

Тогда она определена корректно. 
Единственная возможность улучшить плохие свойства (-a), (-b) и (-c) - это рассмотреть большие группы отражений

$$
W=W_{1} \subset W_{2} \subset \cdots \subset W_{k} \subset \cdots \subset W(S)
$$

с фундаментальными многогранниками $\mathcal{M}_{k}$ и допустимыми множествами $P\left(\mathcal{M}_{k}\right)$ такими, что

$$
P(\mathcal{M})=P\left(\mathcal{M}_{1}\right) \subset P\left(\mathcal{M}_{2}\right) \subset \cdots \subset P\left(\mathcal{M}_{k}\right) \subset \cdots
$$

Это дает возрастающую последовательность лоренцевых алгебр Каца-Муди

$$
\begin{aligned}
\mathfrak{g}^{\prime \prime}\left(A\left(S, W_{1}, P\left(\mathcal{M}_{1}\right)\right)\right) \subset \mathfrak{g}^{\prime \prime} & \left(A\left(S, W_{2}, P\left(\mathcal{M}_{2}\right)\right)\right) \subset \cdots \\
& \cdots \subset \mathfrak{g}^{\prime \prime}\left(A\left(S, W_{k}, P\left(\mathcal{M}_{k}\right)\right)\right) \subset \cdots
\end{aligned}
$$

с одной и той же подалгеброй Картана $M^{*} \otimes \mathbb{C}$. Нетрудно видеть, что эта процедура дает возрастающую последовательность двойственных конусов Титса

$$
T_{1}^{*} \subset T_{2}^{*} \subset \cdots \subset T_{k}^{*} \subset \cdots \subset \overline{V^{+}(S)}
$$

и убывающую последовательность конусов Титса и областей $\Omega\left(S, W_{k}\right)$. Таким образом, по крайней мере плохие свойства (-a) и (-b) улучшаются. K сожалению, эта процедура в общем случае бесконечна и приводит к бесконечно порожденным лоренщевым алгебрам Каца-Муди.

В следующем пункте мы покажем, что, использую эту, может быть, бесконечную процедуру, можно всегда обратить плохие свойства (-a), (-b) и (-c).

2.2. Лоренцевы алгебры Каца-Муди арифметического и ограниченного арифметического типа. Пусть $S: M \times M \rightarrow \mathbb{Z}$-гиперболическая решетка, $W \subset W(S)$ - произвольная группа отражений, $\mathcal{M}$ - фундаментальный многогранник для $W$ и $P(\mathcal{M})$ - допустимое множество ортогональных векторов к $\mathcal{M}$. Как и выше, мы предположим, что $P(\mathcal{M})$ порождает подрешетку конечного индекса в $M$ и имеет связный граф Грама. Используя то же самое определение, что и в п. 2.1, можно определить неразложимую лоренцеву симметризуемую обобщенную матрищу Картана $A=A(S, W, P(\mathcal{M}))$ и лоренцевы алгебры Каца-Муди

$$
\mathfrak{g}^{\prime}(A)=\mathfrak{g}^{\prime}(A(S, W, P(\mathcal{M}))), \quad \mathfrak{g}^{\prime \prime}(A)=\mathfrak{g}^{\prime \prime}(A(S, W, P(\mathcal{M}))) .
$$

Они имеют те же свойства, что и в п. 2.1. Единственное отличие в том, что множество $P(\mathcal{M})$ бесконечно, матрица $A$ бесконечна и множество образуюших $e_{k}, f_{k}, h_{k}$, где $k \in P(\mathcal{M})$, бесконечно. Эта алгебра Ли является объединением ее подалгебр Каца-Муди, определяемых такими конечными множествами $P(\mathcal{M})^{(\leqslant n)} \subset P(\mathcal{M})$, что их элементы имеют высоту $\leqslant \sqrt{n}$ для некоторого фиксированного элемента $h \in M$ такого, что $\mathbb{R}_{++} h \in \mathcal{M}$ и $S(h, h)<0$.

В определении 1.4.1 мы ввели группы отражений $W$ арифметического типа. Применяя это определение к группе Вейля $W$ и множеству простых вешественных корней $P(\mathcal{M})$ алгебры Каца-Муди $\mathfrak{g}^{\prime \prime}(A(S, W, P(\mathcal{M})))$, мы можем использовать его, чтобы ввести лоренцевы алгебры Каца-Муди арифметического типа. Но мы думаем, что для алгебр Каца-Муди $\mathfrak{g}^{\prime \prime}(A(S, W, P(\mathcal{M})))$ более естественным является определение, использующее множество мнимых корней. Мы увидим, что эти две возможности эквивалентны.

Следуюшее определение является обобшением определения из [25], которое было дано для конечно порожденных алгебр Каца-Муди. 
ОПРЕДЕЛЕНИЕ 2.2.1. Лоренцева алгебра Каца-Муди $\mathfrak{g}^{\prime \prime}(A(S, W, P(\mathcal{M})))$ имеет арифметический тип, если для любого $x \in M$ с квадратом $S(x, x)<0$ найдется $n \in \mathbb{N}$ такой, что

$$
n x \in \Delta^{\mathrm{im}} .
$$

Очевидно, это определение эквивалентно равенству открытых рациональных конусов

$$
\mathbb{Q}_{+} \Delta_{+}^{\mathrm{im}} \cap V^{+}(S)=(M \otimes \mathbb{Q}) \cap V^{+}(S) .
$$

Имеем

Теорема 2.2.2. Лоренцева алгебра Каца-Муди $\mathfrak{g}^{\prime \prime}(A(S, W, P(\mathcal{M})))$ имеет арифметический тип, если и только если ее группа Вейля $W$ имеет арифметический тип (таким образом, мы имеем әквивалентные свойства (1.4.3)-(1.4.5), (1.4.9), (1.4.10)). Кроме того, $\mathfrak{g}^{\prime \prime}(A(S, W, P(\mathcal{M})))$ имеет арифметический тип, если и только если конус Титса в существенном совпадает с конусом $V^{+}(S)$, m.e.

$$
\operatorname{Int} T=V^{+}(S), \quad \text { эквивалентно } \quad \bar{T}=\overline{V^{+}(S)} .
$$

ДокАЗАТЕЛЬСтво. Предположим, что $\mathfrak{g}^{\prime \prime}(A(S, W, P(\mathcal{M})))$ имеет арифметический тип. Тогда в силу $(2.2 .2)$ и (2.1.14)

$$
(M \otimes \mathbb{Q}) \cap V^{+}(S) \subset\left(\mathbb{Q}_{+} \Delta_{+}^{\mathrm{im}}\right) \subset T^{*} .
$$

Так как $M \otimes \mathbb{Q}$ всюду плотно в $V^{+}(S)$, отсюда следует, что $V^{+}(S) \subset T^{*}$. Очевидно, $T^{*} \subset \overline{V^{+}(S)}$. Так как ${\overline{V^{+}(S)}}^{*}=\overline{V^{+}(S)}$, получаем $\bar{T}=\overline{V^{+}(S)}$. Отсюда следует равенство (2.2.3). Применяя $(2.2 .2),(2.2 .3)$ и $(2.1 .14)$, получаем $(M \otimes \mathbb{Q}) \cap$ $V^{+}(S) \subset \mathbb{Q}_{+} Q_{+}$, что эквивалентно определению (1.4.3).

Теперь предположим, что группа Вейля $W$ имеет арифметический тип. По (1.4.9) и (1.4.10) тогда имеем

$$
\left(\mathbb{R}_{+} Q_{+}\right)^{*} \subset \overline{V^{+}(S)} \subset \mathbb{R}_{+} Q_{+} .
$$

Отсюда $\quad$ следует, $\quad$ что $\quad \mathbb{R}_{+} K=\left(\mathbb{R}_{+} Q_{+}\right)^{*} \cap \mathbb{R}_{+} Q_{+}=\left(\mathbb{R}_{+} Q_{+}\right)^{*} \cap \overline{V^{+}(S)}=$ $\left(\mathbb{R}_{+} Q_{+}\right)^{*}=\mathbb{R}_{+} \mathcal{M}$, где $\mathcal{M}$ - фундаментальньй многогранник для $W$ в $\mathcal{L}(S)$. Поэтому двойственный конус Титса $T^{*}=W\left(\mathbb{R}_{+} K\right)$ содержит $V^{+}(S)$, и мы получаем равенство (2.2.3). Тогда в силу (2.2.3) и (2.1.14) получаем

$$
\mathbb{Q}_{+} \Delta_{+}^{\mathrm{im}} \cap V^{+}(S)=\mathbb{Q}_{+} Q_{+} \cap V^{+}(S) .
$$

По (1.4.3) имеем $V^{+}(S) \cap(M \otimes \mathbb{Q}) \subset \mathbb{Q}_{+} Q_{+}$. Таким образом, в силу равенства, приведенного вьше, $V^{+}(S) \cap(M \otimes \mathbb{Q}) \subset \mathbb{Q}_{+} \Delta_{+}^{\mathrm{im}}$. По определению $V^{+}(S) \cap\left(\mathbb{Q}_{+} \Delta_{+}^{\mathrm{im}}\right) \subset V^{+}(S) \cap(M \otimes \mathbb{Q})$. Поэтому получаем равенство $(2.2 .2)$, и алгебра Каца-Муди имеет арифметический тип.

В качестве следствия эквивалентных определений (2.2.1)-(2.2.3) получаем 
СлЕДСТвИЕ 2.2.3. Лоренцевы алгебры Каца-Муди $\mathfrak{g}^{\prime \prime}(A(S, W, P(\mathcal{M})))$ арифметического типа - это в точности те лоренцевы алгебры Кача-Муди, которые обладают следующими хорошими свойствами:

(a) рачиональный конус $\mathbb{Q}_{+} \Delta_{+}^{\mathrm{im}}$, порожденный мнимыми корнями, содержит $(M \otimes \mathbb{Q}) \cap V^{+}(S)$;

(b) формула для знаменателя $\Phi(z)$ сходится в естественном комплексифицированном конусе $\Omega(S)=M \otimes \mathbb{R}+i V^{+}(S)$ для больиих $\operatorname{Im} z$ (здесь мьи понимаем сходимость формально, как равенство конусов $\Omega(S, W)=\Omega(S) ;$ в конкретных случаях надо подтвердить эту сходимость подходящими оценками).

На самом деле, (а) и (b) әквивалентныь.

Теперь рассмотрим лоренщевы алгебры Каца-Муди ограниченного арифметического типа. Они вводятся аналогично определению 1.4.2.

ОПРЕДЕЛЕНИЕ 2.2.4. Лоренцева алгебра Каца-Муди $\mathfrak{g}^{\prime \prime}(A(S, W, P(\mathcal{M})))$ имеет ограниченный арифметический тип, если она имеет арифметический тип и группа симметрий $A(P(\mathcal{M})$ ) имеет конечный индекс в $O(S) / W$ (это означает, что соответствующее полупрямое произведение $W . A(P(\mathcal{M}))$ имеет конечный индекс в $O(S))$.

Используя теоремы 1.4.3 и 2.2.2, получаем следующую теорему.

Tеорема 2.2.5. Пусть $S: M \times M \rightarrow \mathbb{Z}$ - гиперболическая решетка, $W \subset W(S)$ - произвольная группа отражений, $\mathcal{M}$ - фундаментальный многогранник для $W, P(\mathcal{M})$ - допустимое множество ортогональных векторов $\kappa \mathcal{M} u$

$$
A(P(\mathcal{M}))=\left\{g \in O_{+}(S) \mid g(P(\mathcal{M}))=P(\mathcal{M})\right\}
$$

- соответствующая группа симметрий.

Тогда лоренцева алгебра Каца-Муди $\mathfrak{g}^{\prime \prime}(A(S, W, P(\mathcal{M})))$ имеет ограниченный арифметический тип, если и только если группа $A(P(\mathcal{M}))$ имеет конечный индекс в $O(S) / W$ (әквивалентно $W . A(P(\mathcal{M}))$ имеет конечный индекс в $O(S))$.

Мы можем переписать формулу для знаменателя (2.1.19) следующим образом:

$$
\frac{\sum_{w \in W} \operatorname{det}(w) e^{-2 \pi i S(w(\rho)-\rho, z)}}{\prod_{\alpha \in \Delta_{+}^{\mathrm{re}}}\left(1-e^{-2 \pi i S(\alpha, z)}\right)^{\operatorname{mult}(\alpha)}}=\prod_{\alpha \in \Delta_{+}^{\mathrm{im}}}\left(1-e^{-2 \pi i S(\alpha, z)}\right)^{\operatorname{mult}(\alpha)} .
$$

Очевидно, что обе части этого равенства инвариантны относительно подгруппы $W \cdot A(P(\mathcal{M}))$. Если алгебра Каца-Муди $\mathfrak{g}^{\prime \prime}(A(S, W, P(\mathcal{M})))$ имеет ограниченньй арифметический тип, то эта подгруппа имеет конечный индекс в арифметической групше $O(S)$.

Таким образом, из вышеприведенных результатов получаем 
СлЕДСТВИЕ 2.2.6. Лоренцевы алгебры Каца-Муди $\mathfrak{g}^{\prime \prime}(A(S, W, P(\mathcal{M})))$ ограниченного арифметического типа - это в точности те лоренцевы алгебры Кача-Муди, которье имеют три хороших свойства:

(a) рациональньй конус $\mathbb{Q}_{+} \Delta_{+}^{\mathrm{im}}$, порожденный мнимыми корнями, содержuт $(M \otimes \mathbb{Q}) \cap V^{+}(S)$;

(b) функция для знаменателя $\Phi(z)$ сходится в естественном комплексифицированном конусе $\Omega(S)=M \otimes \mathbb{R}+i V^{+}(S)$ для больших $\operatorname{Im} z$ (здесь ми понимаем сходимость формально, как равенство конусов $\Omega(S, W)=\Omega(S) ;$ в конкретных случаях надо подтвердить әту сходимость соответствующими оченками);

(c) формула для знаменателя (2.2.5) инвариантна относительно подгруппь W.A $(P(\mathcal{M}))$ конечного индекса в арифметической группе $O(S)$.

На самом деле, свойства (a) и (b) следуют из (c).

Наконец, используя вьшеприведенные результаты и теорему 1.4.7, мы получаем, что всегда существует "правильная" последовательность расширений (2.1.20)-(2.1.23) конечно порожденных лоренщевых алгебр Каца-Муди $\mathfrak{g}^{\prime \prime}\left(A\left(S, W_{k}, P\left(\mathcal{M}_{k}\right)\right)\right)$, которая обрашает плохие свойства $(-\mathrm{a}),(-\mathrm{b})$ и $(-\mathrm{c})$ конечно порожденных лоренщевых алгебр Каца-Муди $\mathfrak{g}^{\prime \prime}\left(A\left(S, W_{k}, P\left(\mathcal{M}_{k}\right)\right)\right)$.

Tеорема 2.2.7. Пусть $S: M \times M \rightarrow \mathbb{Z}$ - әиперболическая решетка, $W_{1} \subset$ $W(S)$ - конечно порожденная группа отражений, $\mathcal{M}_{1}$ - фундаментальный многогранник для $W_{1}$ с конечным допустимым множеством $P\left(\mathcal{M}_{1}\right)$ векторов, ортогональных $\mathcal{M}_{1}$. Пусть $\mathfrak{g}^{\prime \prime}\left(A\left(S, W_{1}, P\left(\mathcal{M}_{1}\right)\right)\right)$ - соответствующая лоренцева алгебра Кача-Муди.

Тогда $W_{1}$ является подгруппой отражений $W_{1} \subset W \subset W(S)$ группь отражений $W$ ограниченного арифметического типа с фундаментальным многогранником $\mathcal{M} \subset \mathcal{M}_{1}$ и допустимым множеством $P(\mathcal{M})$ таким, что $P\left(\mathcal{M}_{1}\right) \subset P(\mathcal{M})$. Это дает вложение

$$
\mathfrak{g}^{\prime \prime}\left(A\left(S, W_{1}, P\left(\mathcal{M}_{1}\right)\right)\right) \subset \mathfrak{g}^{\prime \prime}(A(S, W, P(\mathcal{M})))
$$

конечно порожденной лоренцевой алгебры Кача-Муди $\mathfrak{g}^{\prime \prime}\left(A\left(S, W_{1}, P\left(\mathcal{M}_{1}\right)\right)\right)$ в лоренцеву алгебру Каца-Муди $\mathfrak{g}^{\prime \prime}(A(S, W, P(\mathcal{M})))$ ограниченного арифметического типа (которая имеет хорошие свойства (a), (b) u (c)).

2.3. Лоренцевы алгебры Каца-Муди ограниченного арифметического типа с решеточным вектором Вейля. Вьшеприведенные результаты показывают, что лоренцевы алгебры Каца-Муди ограниченного арифметического типа - это наиболее естественный класс лоренцевых алгебр Каца-Муди, который следует изучать. Однако этот класс огромен. Каждая гиперболическая решетка $S$ ранга $\operatorname{rk} S \geqslant 3$ имеет бесконечно много подгрупп отражений $W \subset W(S)$ ограниченного арифметического типа, которые имеют бесконечный индекс друг относительно друга. Каждая такая группа определяет ее собственные лоренщевы алгебры Каца-Муди ограниченного арифметического типа $\mathfrak{g}^{\prime \prime}(A(S, W, P(\mathcal{M})))$.

$\mathrm{K}$ счастью, сушествует другое естественное ограничение на лоренцевы алгебры Каца-Муди. 
Формула для знаменателя имеет наилучшие и наиболее интересные автоморфные свойства, только если она может быть записана в форме (2.1.15), когда вещественные и мнимые корни рассматриваются вместе. Это можно сделать, только если существует решеточный вектор Вейля $\rho$.

ОПреДЕлЕНИЕ 2.3.1. Лоренцева алгебра Каца-Муди $\mathfrak{g}^{\prime \prime}(A(S, W, P(\mathcal{M})))$ имеет решеточный вектор Вейля, если существует $\rho \in M \otimes \mathbb{Q}$ такой, что

$$
S(\rho, \alpha)=-S(\alpha, \alpha) / 2 \text { для любого } \alpha \in P(\mathcal{M})
$$

Если решеточный вектор Вейля $\rho$ действительно сушествует, мы можем записать формулу для знаменателя в форме (2.1.15), которую мы еще раз здесь выпишем:

$$
\Phi(z)=: \sum_{w \in W} \operatorname{det}(w) e^{-2 \pi i S(w(\rho), z)}=e^{-2 \pi i S(\rho, z)} \prod_{\alpha \in \Delta_{+}}\left(1-e^{-2 \pi i S(\alpha, z)}\right)^{\operatorname{mult}(\alpha)} .
$$

Она антиинвариантна отностительно $W . A(P(\mathcal{M}))$, что означает

$$
\Phi(w \cdot a(z))=\operatorname{det}(w) \Phi(z) \text { для любого } w \in W, \quad a \in A(P(\mathcal{M})) .
$$

В результате получаем следующую теорему.

ТЕОРема 2.3.2. Лорениевы алгебры Кача-Mуди $\mathfrak{g}^{\prime \prime}(A(S, W, P(\mathcal{M})))$ ограниченного арифметического типа с решеточным вектором Вейля - это в точности те лоренцевь алгебры Каца-Муди, которые имеют три хороших свойства:

(а) рациональный конус $\mathbb{Q}_{+} \Delta_{+}^{\mathrm{im}}$, порожденный мнимыми корнями, содержит $(M \otimes \mathbb{Q}) \cap V^{+}(S)$;

(b) функиия для знаменателя $\Phi(z)$ сходится в естественном комплексифицированном конусе $\Omega(S)=M \otimes \mathbb{R}+i V^{+}(S)$ для больиих $\operatorname{Im} z$ (здесь мьь понимаем сходимость формально, как равенство конусов $\Omega(S, W)=\Omega(S) ;$ в конкретных случаях надо подтвердить эту сходимость подходящими оченками);

$(\mathrm{c})+(\mathrm{d})$ формула для знаменателя (2.3.2) антиинвариантна относительно подгруппь $W . A(P(\mathcal{M}))$ конечного индекса в арифметической группе $O(S)$.

Применим результаты п. 1.1 и п. 1.4 к описанию этого наиболее интересного класса лоренцевых алгебр Каца-Муди.

Подобно п. 1.1 дадим

ОПРЕДЕЛЕНИЕ 2.3.2. Пусть $S: M \times M \rightarrow \mathbb{Z}$ - гиперболическая решетка, $W \subset$ $W(S)$ - группа отражений, $\mathcal{M}$ - фундаментальный многогранник для $W, P(\mathcal{M})$ допустимое множество ортогональных векторов к $\mathcal{M}$ и

$$
A(P(\mathcal{M}))=\left\{g \in O_{+}(S) \mid g(P(\mathcal{M}))=P(\mathcal{M})\right\}
$$

- соответствующая группа симметрий. 
Лоренцева алгебра Каца-Муди $\mathfrak{g}^{\prime \prime}(A(S, W, P(\mathcal{M})))$ әллиптична, если $W \subset$ $O(S)$ имеет конечный индекс. В частности, решетка $S$ является әллиптической рефлективной. Лоренцева алгебра Каца-Муди $\mathfrak{g}^{\prime \prime}(A(S, W, P(\mathcal{M})))$ параболична, если $A(P(\mathcal{M}))$ бесконечна, но $W \cdot A(P(\mathcal{M}))$ имеет конечный индекс в $O(S)$ и существует $c \in M$ такой, что $S(c, c)=0$ и $g(c)=c$ для любого $g \in A(P(\mathcal{M}))$ (т.е. $A(P(\mathcal{M}))$ имеет касп $c)$. В частности, решетка $S$ является параболической рефлективной.

Пользуясь теоремами 1.3 .2 и 1.3.3, предложениями 1.4 .10 и 1.4 .11 и результатами п. 2.2 , получаем следующую теорему.

Теорема 2.3.3. Лоренцевы алгебры Каца-Муди $\mathfrak{g}^{\prime \prime}(A(S, W, P(\mathcal{M})))$ ограниченного арифметического типа с решеточным вектором Вейля $\rho$ имеют $S(\rho, \rho) \leqslant 0$, ec $\Omega u \operatorname{rk} S \geqslant 3$.

Для $S(\rho, \rho)<0$ әто в точности әллиптические лоренцевы алгебры Каца-Муди с решеточным вектором Вейля. Их число конечно.

Для $S(\rho, \rho)=0$ это в точности параболические алгебры Кача-Муди с решеточныцм вектором Вейля. Для фиксированного $D>0$ их число конечно, если

$$
\left[O(S)_{\rho}: A(P(\mathcal{M}))\right]<D .
$$

По-видимому, существование обобшенного решеточного вектора Вейля $\rho$ (см. определение 1.4.9) также очень важно для автоморфных свойств формулы для знаменателя (2.3.2) лоренцевых алгебр Каца-Муди. (Формула для знаменателя (2.3.2) хорошо определена после расширения решетки $M$ при помоши конечномерного подпространства в ядре матрицы Грама множества $P(\mathcal{M})$.)

По теоремам 1.1.1 и 1.1.3, предложениям 1.4 .10 и 1.4.11 и результатам п. 1.3 .2 получаем следующую теорему.

ТЕорема 2.3.4. Лоренцевы алгебры Каца-Муди $\mathfrak{g}^{\prime \prime}(A(S, W, P(\mathcal{M})))$ ограниченного арифметического типа с обобщенным решеточным вектором Вейля $\rho$ имеют $S(\rho, \rho) \leqslant 0$, если $\mathrm{rk} S \geqslant 3$.

Для $S(\rho, \rho)<0$ это в точности әллиптические лоренчевы алгебры Кача-Муди. Число их решеток $S: M \times M \rightarrow \mathbb{Z}$ конечно.

Для $S(\rho, \rho)=0$ это в точности әллиптические или параболические лоренчевы алгебры Каца-Муди. Число их решеток $S: M \times M \rightarrow \mathbb{Z}$ также конечно.

ЗАмечАние 2.3.5. Данные результаты показывают, что классификация эллиптических и параболических рефлективных решеток имеет исключительную важность для теории лоренщевых алгебр Каца-Муди. Много эллиптических рефлективных решеток было построено Э.Б. Винбергом ([30]-[34]). В [16]-[18], [22] были классифицированы все эллиптические 2-рефлективные четные гиперболические решетки. Здесь $S$ эллиптична 2-рефлективна, если группа $W^{(2)}(S)$, порожденная отражениями во всех элементах $\delta \in M$ с квадратом $S(\delta, \delta)=2$, имеет конечньй индекс в $O(S)$. В [17] имеется серия из двенадцати 2-элементарных (т.е. с 2 -элементарной дискриминантной группой $M^{*} / M$ ) параболических 2-рефлективных четных гиперболических решеток. Эту серию продолжает четная унимодулярная гиперболическая решетка ранга 26 , параболичность которой доказана 
Дж. Конвеем [7]. В работе Р. Борчердса [1] имеются некоторые примеры эллиптических и параболических рефлективных решеток. В работе О.П. Рузманова [28] описаны некоторые параболические подгруппы отражений групп Бьянки (они действуют в трехмерном пространстве Лобачевского). (См. обзоры Э.Б. Винберга, О.В. Шварцмана [35] и Р. Шарлау и К. Вальхорн [29] по поводу дальнейших результатов по рефлективным решеткам.)

Мы должны сказать, что несмотря на результаты конечности (см. §1), точное описание всех рефлективных гиперболических решеток является трудной проблемой. С другой стороны, в настоящее время известно множество реф̆ективных гиперболических решеток, и в силу результатов конечности все эти примеры очень интересны.

2.4. Комментарий по поводу недавних результатов Р. Борчердса. Здесь мы хотим представить наше понимание недавних результатов Р. Борчердса [2]-[6] в связи с полученными выше результатами.

Рассмотрим лоренцеву алгебру Каца-Муди $\mathfrak{g}^{\prime \prime}(A(S, W, P(\mathcal{M})))$ ограниченного арифметического типа с решеточным вектором Вейля $\rho$ (в силу п. 2.3 эта алгебра эллиптична или параболична). Тогда ее функция знаменателя $\Phi(z)$ определена в комплексифицированном конусе $V^{+}(S)$, равном

$$
\Omega(S)=M \otimes \mathbb{R}+i V^{+}(S),
$$

и сходится для $\operatorname{Im} z \gg 0$.

Мы обозначим через $U: N \times N \rightarrow \mathbb{Z}$ четную унимодулярную решетку сигнатуры $(1,1)$, где $N=\mathbb{Z} e_{1} \oplus \mathbb{Z} e_{2}$ и $U\left(e_{1}, e_{1}\right)=U\left(e_{2}, e_{2}\right)=0, U\left(e_{1}, e_{2}\right)=-1$.

Рассмотрим решетку

$$
S^{\prime}=S \oplus U(k): M^{\prime} \times M^{\prime} \rightarrow \mathbb{Z}, \quad k \in \mathbb{N},
$$

где $M^{\prime}=M \oplus N$. Решетка $S^{\prime}$ определяет область типа IV, которая является одной из двух связных компонент области

$$
\Omega\left(S^{\prime}\right)=\left\{\mathbb{C} \omega \subset M^{\prime} \otimes \mathbb{C} \mid S^{\prime}(\omega, \omega)=0, \quad S^{\prime}(\omega, \bar{\omega})<0\right\} .
$$

Мы нормализуем $\omega \in \mathbb{C} \omega \in \Omega\left(S^{\prime}\right)$ условием $S^{\prime}\left(\omega, e_{1}\right)=-1$. Тогда

$$
\omega=z \oplus(S(z, z) / 2) e_{1} \oplus(1 / k) e_{2}, \quad z \in \Omega(S) .
$$

Это дает вложение

$$
\Omega(S) \subset \Omega\left(S^{\prime}\right),
$$

которое называется вложением, определяемом каспом $e_{1}$.

Мы понимаем недавние результаты Р. Борчердса следуюшим образом.

Наиболее интересные лоренцевы алгебры Каца-Муди $\mathfrak{g}^{\prime \prime}(A(S, W, P(\mathcal{M})))$ ограниченного арифметического типа с решеточным вектором Вейля $\rho$ (таким образом, они являются әллиптическими или параболическими) имеют 
коррекцию обобщенной лоренцевой алгеброй (или, может быть, супералгеброй) Каца-Муди

$$
\mathfrak{g}^{\prime \prime}(A(S, W, P(\mathcal{M}))) \subset \mathfrak{g}^{\prime \prime}(A(S, W, P(\mathcal{M}) \cup H))
$$

такой, что функиия для знаменателя $\widetilde{\Phi}(z)$ корректирующей обобщенной алгебры Каца-Муди $\mathfrak{g}^{\prime \prime}(A(S, W, P(\mathcal{M}) \cup H))$ является автоморфной формой относительно подходящей подгруппы конечного индекса в $O\left(S^{\prime}\right)$ для некоторо$20 \mathrm{k}$.

Естественно называть эту коррекцию коррекичей автоморфной формой или автоморфной коррекиией. Заметим, что априори функция $\Phi(z)$ является антиинвариантной относительно подгруппы конечного индекса $M_{\rho}^{*} .(W \cdot A(P(\mathcal{M})))$ стационарной подгрупшы $O\left(S^{\prime}\right)_{e_{1}}$ каспа. Здесь $M_{\rho}^{*}=\left\{x \in M^{*} \mid S(\rho, x) \in \mathbb{Z}\right\}$.

Более точно это означает, что сушествует автоморфная форма $\widetilde{\Phi}(z)$, которая автоморфна относительно подгрупшы конечного индекса $G \subset O\left(S^{\prime}\right)^{+}$, содержащей $W \cdot A(P(\mathcal{M}))$, является антиинвариантной относительно $W \cdot A(P(\mathcal{M}))$ и имеет разложение Фурье со свойствами, которые мы опишем ниже. Это разложение Фурье имеет вид

$$
\widetilde{\Phi}(z)=\sum_{w \in W} \operatorname{det}(w)\left(e^{-2 \pi i S(w(\rho), z)}-\sum_{a \in M_{P(\mathcal{M})}^{*} \cap \mathbb{R}_{++} \mathcal{M}} m(a) e^{-2 \pi i S(w(\rho+a), z)}\right),
$$

где все $m(a) \in \mathbb{Z}$ и $m(a) \in \mathbb{Z}_{+}$(в случае супералгебры $\left.m(a) \in \mathbb{Z}\right)$, если $S(a, a)<0$. (См. определение $M_{P(M)}^{*}$ в (1.2.4).) Рассмотрим теперь те $a \in M_{P(\mathcal{M})}^{*} \cap \mathbb{R}_{++} \mathcal{M}$, для которых $S(a, a)=0$. Такие $a$ образуют несколько изотропных лучей, соответствуюших бесконечно удаленным вершинам $\mathcal{M}$ и каспу $\mathcal{M}$, если $\mathcal{M}$ параболичен. Рассмотрим любой из таких лучей $t a_{0}$, где $a_{0}$-примитивный элемент $M_{P(M)}^{*} \mathrm{c}$ квадратом $S\left(a_{0}, a_{0}\right)=0$ и $t \in \mathbb{N}$. Для каждого из таких изотропных лучей должно выполняться равенство для формальных степенных рядов от одной переменной $q$

$$
1-\sum_{t \in \mathbb{N}} m\left(t a_{0}\right) q^{t}=\prod_{k \in \mathbb{N}}\left(1-q^{k}\right)^{\tau\left(k a_{0}\right)}
$$

где $\tau\left(k a_{0}\right) \in \mathbb{Z}_{+}$(в случае супералгебры $\left.\tau\left(k a_{0}\right) \in \mathbb{Z}\right)$.

Рассмотрим

$$
\begin{aligned}
H=\left\{m(a) a \mid a \in M_{P(\mathcal{M})}^{*} \cap \mathbb{R}_{++} \mathcal{M} \text { и } S(a, a)<0\right\} \cup \\
\cup\left\{\tau(a) a \mid a \in M_{P(\mathcal{M})}^{*} \cap \mathbb{R}_{++} \mathcal{M} \text { и } S(a, a)=0\right\},
\end{aligned}
$$

где $k a$ означает, что мы повторяем каждьй элемент $a$ ровно $k$ раз (берем с кратностью $k$ ). Рассмотрим матрицу Грама

$$
G(P(\mathcal{M}) \cup H)
$$


элементов $P(\mathcal{M}) \cup H$. Легко видеть, что $G(P(\mathcal{M}) \cup H)$ имеет все необходимые свойства, чтобы задать обобщенную алгебру Каца-Муди

$$
\mathfrak{g}^{\prime \prime}(G(S, W, P(\mathcal{M}) \cup H)),
$$

соответствующую этой матрице (см. [2] по поводу этого определения). Эта алгебра имеет свойства, подобные свойствам лоренцевых алгебр Каца-Муди, которые мы рассматривали в пп. 2.1 и 2.2. Единственное отличие заключается в том, что множеством простых корней является теперь $P(\mathcal{M}) \cup H$. Эта алгебра градуирована решеткой $M_{P(\mathcal{M})}^{*} \supset M$, имеет корни, кратности корней и ту же самую группу Вейля $W$. Алгебра Каца-Муди $\mathfrak{g}^{\prime \prime}(A(S, W, P(\mathcal{M})))$ задается матрицей Грама $G(P(\mathcal{M}))$ элементов $P(\mathcal{M})$ и является подалгеброй $\mathfrak{g}^{\prime \prime}(G(S, W, P(\mathcal{M}) \cup H))$. Формула для знаменателя алгебры $\mathfrak{g}^{\prime \prime}(G(S, W, P(\mathcal{M}) \cup H))$ равна

$$
\widetilde{\Phi}(z)=e^{-2 \pi i S(\rho, z)} \prod_{\alpha \in \Delta_{+}}\left(1-e^{-2 \pi i S(\alpha, z)}\right)^{\operatorname{mult}(\alpha)},
$$

где $\widetilde{\Phi}(z)$ - функция, описанная в (2.4.5)-(2.4.7) (см. [3], где фактически требуемые вычисления были проделаны). В частности, если сушествует автоморфная форма $\widetilde{\Phi}(z)$, которая описана выше, то она автоматически имеет формулу произведения (2.4.10).

Следуюший наиболее удивительный пример "коррекции" был найден Р. Борчердсом [3], [5]. Рассмотрим четную унимодулярную гиперболическую решетку $S$ сигнатуры $(25,1)$ и $W=W(S)$. Эта группа отражений параболична и имеет решеточный вектор Вейля $\rho$ с квадратом $S(\rho, \rho)=0$. Это было доказано Дж.Конвеем [7]. Возьмем $P(\mathcal{M})=P(\mathcal{M})_{\mathrm{pr}}$ для фундаментального многогранника $\mathcal{M}$ группы $W$. Откорректированная формула для знаменателя этой алгебры Ли равна

$$
\begin{aligned}
\widetilde{\Phi}(z) & =\sum_{w \in W} \operatorname{det}(w) \sum_{n>0} \tau(n) e^{-2 \pi i n(w(\rho), z)} \\
& =e^{-2 \pi i S(\rho, z)} \prod_{\alpha \in \Delta_{+}}\left(1-e^{-2 \pi i S(\alpha, z)}\right)^{p_{24}(1-S(\alpha, \alpha) / 2)} .
\end{aligned}
$$

Здесь

$$
\sum_{n \geqslant 0} \tau(n) q^{n}=q \prod_{n>0}\left(1-q^{n}\right)^{24}
$$

И

$$
\sum_{n \geqslant 0} p_{24}(n) q^{n}=\prod_{n>0}\left(1-q^{n}\right)^{-24} .
$$

Таким образом, в этом случае $H=\rho, 2 \rho, \ldots, t \rho, \ldots$, где каждый элемент $t \rho$ берется с кратностью 24 . Функция $\widetilde{\Phi}(z)$ является автоморфной формой веса 12 относительно подгруппы $O(S \oplus U)^{+} \subset O(S \oplus U)$, которая сохраняет связные компоненты области $\Omega(S \oplus U)$ (см. другие примеры в [5], [6] (а также [3], [4]) и [12]).

По-видимому, основой для построения подобных примеров и многих других является арифметический подъем форм Якоби на области IV-го типа, построенный 
В. А. Гриценко [10], [11]. Некоторый его мультипликативный аналог построен Р. Борчердсом [5].

Теория автоморфных форм - это очень деликатная область. Автоморфные формы, подобные (2.4.11), вероятно, очень редки. С другой стороны, как мы показали вьше, в сушественном имеется только конечное число эллиптических и параболических лоренцевых алгебр Каца-Муди с решеточным вектором Вейля. Таким образом, в существенном следует откорректировать только конечное число лоренцевых алгебр Каца-Муди. Может быть, это возможно, как и для вышеприведенного примера.

\section{Список литературы}

1. Borcherds R.E. Lattices like the Leech lattice // J. Algebra. 1990. V. 130. P. 219-234.

2. Borcherds R.E. Generalized Kac-Moody algebras // J. Algebra. 1988. V. 115. P. 501-512.

3. Borcherds R.E. The monster Lie algebra // Adv. Math. 1990. V. 83. P. 30-47.

4. Borcherds R.E. The monstrous moonshine and monstrous Lie superalgebras // Inv. math. 1992. V. 109. P. 405-445.

5. Borcherds R.E. Automorphic forms on $O_{s+2,2}(\mathbb{R})$ and infinite products // Inv. math. 1995. V. 120. P. 161-213.

6. Borcherds R.E. The moduli space of Enriques surfaces and the fake monster Lie superalgebra / Preprint University of California at Berkeley, 1994.

7. Conway J.H. The automorphism group of the 26 dimensional even Lorentzian lattice // J. Algebra. 1983. V. 80. P. 159-163.

8. Esselmann F. Über die maximale Dimension von Lorentz-Gittern mit coendlicher Spiegelungsgruppe // Universität Bielefeld. Sonderforschungsbereich 343. Preprint, 1992. № 1992-23.

9. Greenberg L. Finiteness theorems for Fuchsian and Kleinian groups // Discrete groups and automorphic functions / Ed. W.J. Harvey. London: Acad. Press, 1977. P. 199-257.

10. Грищенко B.A. Функции Якоби от $n$ переменных // Зап. науч. семин. ЛОМИ. 1988. T. 168 . C. $32-45$.

11. Гриченко B.A. Модулярные формы и пространства модулей абелевых поверхностей и поверхностей К3 // Алгебра и анализ. 1994. Т. 66. С. 65-102.

12. Gritsenko V.A., Nikulin V.V. Siegel automorphic form correction of some Lorentzian Kac-Moody Lie algebras // Matematica. Gottingensis Schriftenreihe des Sonderforschungsbereichs Geometrie und Analysis. 1995. Heft 17. P. 1-26; or Duke e-prints, alg-geom/9504006.

13. Kac V. Infinite dimensional Lie algebras. Cambridge Univ. Press, 1990.

14. Хованский А.Г. Гиперплоские сечения многогранников, торические многообразия и дискретные группы в пространствах Лобачевского // Функц. анализ и его прилож. 1986. T. 20 . C. $50-61$.

15. Kovács S.J. The cone of curves of a K3 surface/ Preprint University of Utah, 1993.

16. Никулин B.B. О факторгруппах групп автоморфизмов гиперболических форм по подгруппам, порожденным 2-отражениями // ДАН СССР. 1979. Т. 248. С. $1307-1309$.

17. Никулин B.В. О факторгруппах групп автоморфизмов гиперболических форм по подгруппам, порожденным 2-отражениями. Алгебро-геометрические приложения // Современные проблемы математики. Итоги науки и техники. М.: ВИНИТИ, 1981. С. 3-114.

18. Никулин В.В. Поверхности типа К3 с конечной группой автоморфизмов и группой Пикара ранга три // Тр. МИАН. 1984. Т. 165. С. 119-142.

19. Никулин В.В. Об арифметических группах, порожденных отражениями, в пространствах Лобачевского // Изв. АН СССР. Сер. матем. 1980. Т. 44. № 3. С. 637-668.

20. Никулин B.В. О классификации арифметических групп, порожденных отражениями, в пространствах Лобачевского // Изв. АН СССР. Сер. матем. 1981. Т. 45. № 1. С. 113-142. 
21. Никулин В.В. Об описании групп автоморфизмов поверхностей Энриквеса // ДАН CCCP. 1984. T. 277. C. $1324-1327$.

22. Nikulin V.V. Discrete reflection groups in Lobachevsky spaces and algebraic surfaces // Proc. Int. Congr. Math. Berkeley. 1986. V. 1. P. 654-669.

23. Nikulin $V . V$. Algebraic surfaces with log-terminal singularities and nef anticanonical class and reflection groups in Lobachevsky spaces / Preprint/Max-Planck-Institut für Mathematik, 1989. MPI/89-28.

24. Nikulin V.V. Basis of the diagram method for generalized reflection groups in Lobachevsky spaces and algebraic surfaces with nef anticanonical class // Int. J. Math. (в печати).

25. Nikulin V.V. A lecture on Kac-Moody Lie algebras of the arithmetic type. Queen's University, 1994. Preprint \#1994-16; or Duke e-prints, alg-geom/9412003.

26. Nikulin V.V. Reflection groups in hyperbolic spaces and the denominator formula for Lorentzian Kac-Moody Lie algebras // Matematica Gottingensis Schriftenreihe des Sonderforschungsbereichs Geometrie und Analysis. 1995. Heft 13. P. 1-30; or Duke e-prints, alg-geom/9503003.

27. Прохоров М.Н. Отсутствие дискретных групп отражений с некомпактным фундаментальным многогранником конечного объема в пространствах Лобачевского большой размерности // Изв. АН СССР. Сер. матем. 1986. Т. 50. № 2. С. 413-424.

28. Рузманов О.П. Подгруппы отражений в группах Бьянки // УМН. 1990. Т. 45. № 1 . C. $189-190$.

29. Scharlau $R$., Walhorn $C l$. Integral lattices and hyperbolic reflection groups // Astérisque. 1992. V. 209. P. 279-291.

30. Винберг Э.Б. Дискретные группы, порожденные отражениями, в пространствах Лобачевского // Матем. сб. 1967. Т. 72. С. 471-488.

31. Винберг Э.Б. О группах единиц некоторых квадратичных форм // Матем. сб. 1972. T. 87. C. $18-36$.

32. Винберг Э.Б. Отсутствие кристаллографических групп отражений в пространствах Лобачевского большой размерности // Тр. Моск. матем. об-ва. 1984. Т. 47. С. 68-102.

33. Винберг Э.Б. Гиперболические группы отражений // УМН. 1985. Т. 40. С. 29-66.

34. Vinberg É.B. Discrete reflection groups in Lobachevsky spaces // Proc. Int. Congr. Math. Warsaw. 1983. P. 593-601.

35. Винберг Э.Б., Швариман О.В. Дискретные группы движений пространств постоянной кривизны // Геометрия 2. Совр. пробл. матем. Фундам. напр. Т. 29. М.: ВИНИТИ, 1988. C. $147-259$.

Математический институт им. В.А. Стеклова РАН, slava@nikulin.mian.su 